\title{
The Numerical Solution of Fractional Black-Scholes-Schrodinger Equation Using the RBFs Method
}

\author{
Naravadee Nualsaard, ${ }^{1}$ Anirut Luadsong $\mathbb{D}^{1,2}$ and Nitima Aschariyaphotha $\mathbb{D}^{2}$ \\ ${ }^{1}$ Department of Mathematics, Faculty of Science, King Mongkut's University of Technology Thonburi (KMUTT), 126 Bang Mod, \\ Thung Khru, Bangkok 10140, Thailand \\ ${ }^{2}$ Ratchaburi Learning Park, King Mongkut's University of Technology Thonburi (KMUTT), Rang Bua, Chom Bueng, \\ Ratchaburi 70150, Thailand \\ Correspondence should be addressed to Anirut Luadsong; anirut.lua@kmutt.ac.th
}

Received 7 November 2019; Revised 13 February 2020; Accepted 10 March 2020; Published 15 May 2020

Academic Editor: Stephen C. Anco

Copyright (c) 2020 Naravadee Nualsaard et al. This is an open access article distributed under the Creative Commons Attribution License, which permits unrestricted use, distribution, and reproduction in any medium, provided the original work is properly cited.

\begin{abstract}
In this paper, radial basis functions (RBFs) method was used to solve a fractional Black-Scholes-Schrodinger equation in an option pricing of financial problems. The RBFs method is applied in discretizing a spatial derivative process. The approximation of time fractional derivative is interpreted in the Caputo's sense by a simple quadrature formula. This RBFs approach was theoretically proved with different problems of two numerical examples: time step arbitrage bubble case and time linear arbitrage bubble case. Then, the numerical results were compared with the semiclassical solution in case of fractional order close to 1 . As a result, both numerical examples showed that the option prices from RBFs method satisfy the semiclassical solution.
\end{abstract}

\section{Introduction}

An option is one of the most important and popular financial derivatives in financial market. There are various types of mathematical model for option pricing. The Black-Scholes equation, introduced by Black and Scholes [1], provided an approximate description of underlying asset price behavior. This equation becomes popular in various kinds of studies such as economics, physics, and financial mathematics since it can be simply solved with a short time in conversion into the solutions. The Black-Scholes equation is a well-known financial model in option pricing which is constructed under strict assumptions. In fact, arbitrage exists in real financial markets; however, one of the key assumptions in this equation has no arbitrage. Thus, Classical Black-Scholes equation was extended for arbitrage possibilities by Contreras et al. [2] . The Black-Scholes equation with arbitrage can be interpreted using quantum mechanic's view point in a sense of an imaginary time from Schrodinger equation of a free particle. Therefore, the Black-Scholes equation including arbitrage possibilities was proposed by Contreras et al. [3] which the equation was solved by the semiclassical method. Although the Black-Scholes-Schrodinger equation can be used to describe the analysis of option pricing in financial markets, this equation cannot be completely described in the physical meaning of the actual financial market.

For a decade, fractional differential equations (FDEs) have been further used as a tool to describe the phenomena in applied sciences and engineering. Problems related to biology, chemistry, physics, mechanics, and engineering (e.g., surface and subsurface hydrology [4-6], finance [7, 8], epidemiology [9], and ecology $[10,11])$ were efficiently explained by fractional differential and integral equations. As a result, FDEs are more suitably compared to the integer-order models [12]. Moreover, the fractional derivative can be used to describe some occurrences that integer cannot. There are many different types of fractional derivative. A most popular of fractional derivatives was proposed by Caputo [13] that it was deeply explained referred to Oldham and Spanier [14], Miller and Ross [15], Podlubny [16], and Kilbas et al. [17]. Sometimes, FDEs are not suitable for some analytical methods; the numerical methods that contribute to an 
abundance of approaches are used to overcome this disadvantage. Finite difference and meshless method are commonly applied in numerical methods. Cen and Le [18] presented a numerical method based on central difference spatial discretization for a generalized Black-Scholes equation for option pricing. Song and Wang [19] also solved the put option pricing problem based on the fractional BlackScholes equation by finite difference method. The results proved that applications of this technique are efficiency and less requirement for computational work to solve the fractional Black-Scholes equation. Kumar et al. [20] provide the numerical algorithm called homotopy perturbation and homotopy analysis method for time fractional BlackScholes equation to solve a European option problem. Phaochoo et al. [21, 22] proposed a numerical method based on the meshless local Petrov-Galerkin (MLPG) to solve a Black-Scholes equation and fractional Black-Scholes equation via moving kriging interpolation for financial problems. Likewise, Phramrung et al. [23] applied the numerical method of meshless local Petrov-Galerkin (MLPG) to approximate the problem of the fractional HIV model. In addition, Cen et al. [24] applied the central difference spatial discretization for time fractional Black-Scholes equation. Chen et al. [25] introduced a new operator splitting method for numerical approach in an American option under fractional Black-Scholes model. Numerical scheme was also operated by Uddin and Taufiq [26] using radial kernels and Laplace transform to approximate the time fractional Black-Scholes model governing European options.

Radial basis functions (RBFs), firstly introduced in 1971, is a new technique for the numerical solution of partial differential equations (PDEs) in Hardy research [27]. The RBFs are high-dimensional and highly accurate meshless computational algorithm with a number of distinct advantages. It is widely applied in field of applied science and engineering such as diffusion equations [28], reconstruction of corrupted images [29, 30], and surface reconstruction [31]. The generation of a grid impacted is not required in the RBFs method because it is particularly efficient in solving such kind of free boundary and convection dominated problems [32]. Furthermore, the RBFs approximation technique is based on collocation in a set of scattered nodes. This method is independent with respect to the dimension of the space. However, there are few studies that applied the RBFs method in option pricing. Hon and Mao [33] proposed a radial basis functions (RBFs) method for solving options pricing model with RBFs interpolation by converting Black-Scholes equation into a system of ordinary differential equations (ODEs). Besides, Hon [34] combined the quasi-interpolation and RBFs method, called a quasi-radial basis function method, to solve the option pricing model. The result showed a high accuracy in the computations for European and American options. Zhang [35] applied a radial basis functions method for valuing options with multinomial tree approach. The study claimed that the RBFs method is highly efficient for both European option and American option.

In this study, the fractional Black-Scholes-Schrodinger equation is solved by using RBFs method for an option pricing. Spatial derivative was discretized through this method, and the approximation of time fractional derivative is interpreted in the Caputo's senses by a simple quadrature formula. Then, numerical solution is compared with the semiclassical solution. The procedure in this study was demonstrated in Section 2, where the basic concept of the BlackScholes-Schrodinger equation was briefly described as a problem formulation. Spatial discretization of the fractional Black-Scholes-Schrodinger equation was analyzed by using the RBFs method as it was shown in Section 3, and temporal discretization of fractional Black-Scholes-Schrodinger equation was investigated with a simple quadrature formula in Section 4 . Then, the RBFs method was confirmed by stability analysis to ensure the suitability of this method in Section 5. After that, in Section 6, numerical solutions were examined and discussed to validate the proposed method to summarize the conclusion of this work in Section 7.

\section{Problem Formulation}

The Black-Scholes-Schrodinger equation is a quantum financial model which is used for analyzing fair prices of options in real financial market. This equation interprets the BlackScholes equation with arbitrage possibilities in quantum mechanic's view point in the senses of the Schrodinger equation. The Black-Scholes equation with arbitrage possibilities is transformed to the Black-Scholes-Schrodinger equation. The Black-Scholes equation with arbitrage possibilities in the domain $(S, t) \in \mathbb{R} \times[0, T]$ is presented in the form of Equation (1).

$$
\frac{\partial \pi}{\partial t}+\frac{\sigma^{2}}{2} S^{2} \frac{\partial^{2} \pi}{\partial S^{2}}+r \frac{(\sigma-(\bar{\alpha} f / r))}{(\sigma-f)}\left(S \frac{\partial \pi}{\partial S}-\pi\right)=0,
$$

where $\pi(S, t)$ is the option price at underlying asset price $S$ with time $t, S$ represents an underlying asset price, $t$ represents time variable, $\sigma$ represents the volatility of underlying asset price, $r$ is the risk free interest rate, $T$ is the expiration date, and the $f(t)$ is called the arbitrage bubble function. In case of $f=0$, Equation (1) is reduced to the original BlackScholes equation with arbitrage possibilities. Consequently, the degeneration will occur in approximation when $S$ converges to 0 . In order to solve this problem, the changing variable technique is applied by $\xi=\ln S$, to obtain

$\frac{\partial \pi}{\partial t}+\frac{\sigma^{2}}{2} \frac{\partial^{2} \pi}{\partial \xi^{2}}+\left(r-\frac{\sigma^{2}}{2}\right) \frac{\partial \pi}{\partial \xi}+\frac{(r-\bar{\alpha}) f}{\sigma-f}\left(\frac{\partial \pi}{\partial \xi}-\pi\right)-r \pi=0$.

In 2010, a new variable, $x=\xi-\left(r-\left(\sigma^{2} / 2\right)\right) t$ and $\pi(x, t)=e^{-r(T-t)} \psi(x, t)$, is first introduced by Contreras et al. [3]. Therefore, Equation (2) transforms to the Black-Scholes-Schrodinger equation in the domain $(x, t)$ $\in \mathbb{R} \times[0, T]$ as follows:

$$
\frac{\partial \psi(x, t)}{\partial t}+\frac{\sigma^{2}}{2} \frac{\partial^{2} \psi(x, t)}{\partial x^{2}}+v(x, t)\left(\frac{\partial \psi(x, t)}{\partial x}-\psi(x, t)\right)=0,
$$


where $\psi(x, t)$ represents a wave function at time $t, v(x, t)$ is a potential function, $v(x, t)=(r-\bar{\alpha}) \check{f}(x, t) /(\sigma-\check{f}(x, t))$, $\check{f}(x, t)=f\left(e^{x+\left(r-\left(\sigma^{2} / 2\right)\right) t}, t\right)$, and $x$ is called a space variable.

However, the Black-Scholes-Schrodinger equation is not completely consistent with the actual financial market. Therefore, the fractional calculus is used to apply in the Black-Scholes-Schrodinger equation to describe occurrences in financial market especially in field of log-price probability and to specify the variability in prices. The fractional Black-Scholes-Schrodinger equation in the domain $(x, t)$ $\in \mathbb{R} \times[0, T]$ can be expressed as

$$
\frac{\partial^{\alpha} \psi(x, t)}{\partial t^{\alpha}}+\frac{\sigma^{2}}{2} \frac{\partial^{2} \psi(x, t)}{\partial x^{2}}+v(x, t)\left(\frac{\partial \psi(x, t)}{\partial x}-\psi(x, t)\right)=0,
$$

where $\alpha$ is a fractional order, $0<\alpha \leq 1$. It is also considered as a model parameter, in which each model will provide its solution. Equation (4) is reduced to the original Black-Scholes-Schrodinger equation when $\alpha=1$. Furthermore, this study investigates the solution in each different value of $\alpha$, where it can be any number in $(0,1]$ and examine how it affects the solution of the model.

\section{Spatial Discretization}

In this section, the radial basis functions (RBFs) method is applied in the process of discretizing a space variable, because the RBFs interpolation is stable and accurate [36]. The RBFs interpolation formulation can be written as

$$
\psi(x, t)=\sum_{j=1}^{N_{x}} R_{j}(x) \beta_{j}(t)
$$

where $R_{j}(x)$ is the RBFs, $\beta_{j}(t)$ is an unknown coefficient at time, $t$, and $N_{x}$ is the number of support nodes in the interpolation domain of point $x$.

Firstly, Equation (5) is substituted into the fractional Black-Scholes-Schrodinger equation (Equation (4)).

$$
\begin{aligned}
\frac{\partial^{\alpha}}{\partial t^{\alpha}}\left(\sum_{j=1}^{N_{x}} R_{j}(x) \beta_{j}(t)\right) & +\frac{\sigma^{2}}{2} \frac{\partial^{2}}{\partial x^{2}}\left(\sum_{j=1}^{N_{x}} R_{j}(x) \beta_{j}(t)\right) \\
& +v(x, t) \frac{\partial}{\partial x}\left(\sum_{j=1}^{N_{x}} R_{j}(x) \beta_{j}(t)\right) \\
& -v(x, t) \sum_{j=1}^{N_{x}} R_{j}(x) \beta_{j}(t)=0,
\end{aligned}
$$

$$
\begin{aligned}
\sum_{j=1}^{N_{x}} R_{j}(x) \frac{d^{\alpha} \beta_{j}(t)}{d t^{\alpha}} & +\frac{\sigma^{2}}{2} \sum_{j=1}^{N_{x}} R_{j, x x}(x) \beta_{j}(t) \\
& +v(x, t) \sum_{j=1}^{N_{x}} R_{j, x}(x) \beta_{j}(t) \\
& -v(x, t) \sum_{j=1}^{N_{x}} R_{j}(x) \beta_{j}(t)=0, \\
\sum_{j=1}^{N_{x}} R_{j}(x) \frac{d^{\alpha} \beta_{j}(t)}{d t^{\alpha}} & +\sum_{j=1}^{N_{x}}\left(\frac{\sigma^{2}}{2} R_{j, x x}(x)+v(x, t) R_{j, x}(x)\right. \\
& \left.-v(x, t) R_{j}(x)\right) \beta_{j}(t)=0,
\end{aligned}
$$

where $R_{j, x}(\cdot)=\partial R_{j}(\cdot) / \partial x$ and $R_{j, x x}(\cdot)=\partial^{2} R_{j}(\cdot) / \partial x^{2}$ for each node $x_{i}, i=1,2, \cdots, N_{x}$

$$
\begin{aligned}
\sum_{j=1}^{N_{x}} R_{j}\left(x_{i}\right) \frac{d^{\alpha} \beta_{j}(t)}{d t^{\alpha}} & +\sum_{j=1}^{N_{x}}\left(\frac{\sigma^{2}}{2} R_{j, x x}\left(x_{i}\right)+v\left(x_{i}, t\right) R_{j, x}\left(x_{i}\right)\right. \\
& \left.-v\left(x_{i}, t\right) R_{j}\left(x_{i}\right)\right) \beta_{j}(t)=0 .
\end{aligned}
$$
form as

Secondly, Equation (7) can be written in the matrix

$$
\mathbf{A} \frac{d^{\alpha} \boldsymbol{\beta}(t)}{d t^{\alpha}}+\mathbf{B} \boldsymbol{\beta}(t)=\mathbf{0}
$$

where

$$
\begin{aligned}
& \mathbf{A}=\left[A_{i j}\right]_{N \times N^{\prime}}, A_{i j}=R_{j}\left(x_{i}\right), \\
& \mathbf{B}=\left[B_{i j}\right]_{N \times N} ; B_{i j}=\frac{\sigma^{2}}{2} R_{j, x x}\left(x_{i}\right)+v\left(x_{i}, t\right) R_{j, x}\left(x_{i}\right)-v\left(x_{i}, t\right) R_{j}\left(x_{i}\right), \\
& \boldsymbol{\beta}=\left[\beta_{j}\right]_{N \times 1}=\left[\beta_{1}, \beta_{2}, \cdots, \beta_{N}\right]^{T}, \text { for } i=1,2, \cdots, N_{x} \text { and } j=1,2, \cdots, N_{x} .
\end{aligned}
$$

From a spatial discretization of fractional BlackScholes-Schrodinger equation, the ordinary differential equation system of each point in the space is obtained. Therefore, these systems are in form of time-dependent equation. In the next section, a simple quadrature formula is applied for discretization of time variable.

\section{Temporal Discretization}

In this section, the systems of ODEs from previous section will be discretized on time variable by a simple 
quadrature formula. First of all, the time fractional derivative, $\partial^{\alpha} \psi(x, t) / \partial t^{\alpha}$ in Equation (4) is defined by Caputo's viewpoint of order $\alpha(0<\alpha \leq 1)$ as

$$
\frac{\partial^{\alpha} \psi(x, t)}{\partial t^{\alpha}}=\frac{1}{\Gamma(1-\alpha)} \int_{0}^{t} \frac{\partial \psi(x, \tau)}{\partial \tau}(t-\tau)^{-\alpha} d \tau,
$$

where $\Gamma(\cdot)$ denote the gamma function.

The variable $t_{n}$ is defined as $t_{n}=n \Delta t, n=0,1,2, \cdots, N_{t}$, where $N_{t}$ is a number of time step and $\Delta t=T / N_{t}$ is the step size of a time variable. A simple quadrature formula from [37] is applied as

$$
\frac{d^{\alpha} \boldsymbol{\beta}}{d t^{\alpha}}=\bar{\sigma}_{\alpha, \Delta t} \sum_{j=1}^{n} \omega_{j}^{(\alpha)}\left(\boldsymbol{\beta}^{n-j+1}-\boldsymbol{\beta}^{n-j}\right)+O(\Delta t),
$$

where $\omega_{j}^{(\alpha)}=j^{1-\alpha}-(j-1)^{1-\alpha}$ and $\bar{\sigma}_{\alpha, \Delta t}=(1 / \Gamma(1-\alpha))(1 / 1$ $-\alpha)\left(1 / \Delta t^{\alpha}\right)$.

Hence, $d^{\alpha} \boldsymbol{\beta} / d t^{\alpha}=D_{t}^{(\alpha)} \boldsymbol{\beta}^{\mathrm{n}}+O(\Delta t)$, and the first-order approximation method for the computation of Caputo's fractional derivative is given by

$$
D_{t}^{(\alpha)} \boldsymbol{\beta}^{n}=\bar{\sigma}_{\alpha, \Delta t} \sum_{j=1}^{n} \omega_{j}^{(\alpha)}\left(\boldsymbol{\beta}^{n-j+1}-\boldsymbol{\beta}^{n-j}\right) .
$$

Applying Equation (12) in Equation (8),

$$
\begin{gathered}
\mathbf{A}\left[\bar{\sigma}_{\alpha, \Delta t} \sum_{j=1}^{n} \omega_{j}^{(\alpha)}\left(\boldsymbol{\beta}^{n-j+1}-\boldsymbol{\beta}^{n-j}\right)\right]+\mathbf{B} \boldsymbol{\beta}^{n}=0 \\
\mathbf{A} \bar{\sigma}_{\alpha, \Delta t} \omega_{1}^{(\alpha)}\left(\boldsymbol{\beta}^{n}-\boldsymbol{\beta}^{n-1}\right)=-\mathbf{A} \bar{\sigma}_{\alpha, \Delta t} \sum_{j=2}^{n} \omega_{j}^{(\alpha)}\left(\boldsymbol{\beta}^{n-j+1}-\boldsymbol{\beta}^{n-j}\right)-\mathbf{B} \boldsymbol{\beta}^{n} .
\end{gathered}
$$

For $n=1$, Equation (13) can be rewritten as

$$
\left(\mathbf{A} \bar{\sigma}_{\alpha, \Delta t} \omega_{1}^{(\alpha)}+\mathbf{B}\right) \boldsymbol{\beta}^{1}=\mathbf{A} \bar{\sigma}_{\alpha, \Delta t} \omega_{1}^{(\alpha)} \boldsymbol{\beta}^{0}
$$

and for $n \geq 2$,

$$
\begin{aligned}
\left(\mathbf{A} \bar{\sigma}_{\alpha, \Delta t} \omega_{1}^{(\alpha)}+\mathbf{B}\right) \boldsymbol{\beta}^{n}= & \mathbf{A} \bar{\sigma}_{\alpha, \Delta t} \omega_{1}^{(\alpha)} \boldsymbol{\beta}^{n-1} \\
& -\mathbf{A} \bar{\sigma}_{\alpha, \Delta t} \sum_{j=2}^{n} \omega_{j}^{(\alpha)}\left(\boldsymbol{\beta}^{n-j+1}-\boldsymbol{\beta}^{n-j}\right) .
\end{aligned}
$$

The formula in Equation (15) is applied to approximate at the time level $n=1$ and also used in Equation (16) for $n \geq 2$. Then, Equation (16) is substituted into Equation (5) to obtain the solution at each time level $n$. Equation (16) can be expressed as

$$
\boldsymbol{\beta}^{n}=\mathbf{G}^{n}\left(\boldsymbol{\beta}^{n-1}-\frac{1}{\omega_{1}^{(\alpha)}} \sum_{j=2}^{n} \omega_{j}^{(\alpha)}\left(\boldsymbol{\beta}^{n-j+1}-\boldsymbol{\beta}^{n-j}\right)\right),
$$

where $\mathbf{G}^{n}=\left(\mathbf{I}+\left(\mathbf{A} \bar{\sigma}_{\alpha, \Delta t} \omega_{1}^{(\alpha)}\right)^{-1} \mathbf{B}\right)^{-1}$.

\section{Stability Analysis}

In this section, we analyze the stability of the RBFs method. Let $\mathbf{e}^{n}=\boldsymbol{\beta}^{n}-\tilde{\boldsymbol{\beta}}^{n}$ be a small perturbation at the time level $n$, where $\boldsymbol{\beta}^{n}$ is the exact solution and $\tilde{\boldsymbol{\beta}}^{n}$ is an approximate solution. Therefore, the equation of error $\mathbf{e}^{n}$ can be written as

$$
\mathbf{e}^{n}=\mathbf{G}^{n}\left(\mathbf{e}^{n-1}-\frac{1}{\omega_{1}^{(\alpha)}} \sum_{j=2}^{n} \omega_{j}^{(\alpha)}\left(\mathbf{e}^{n-j+1}-\mathbf{e}^{n-j}\right)\right) .
$$

Equation (17) would be stable if boundary of $n$ in $\mathbf{e}^{n}$ is increased indefinitely by $n$ exists as a positive number, $M$ that $\left\|\mathbf{G}^{n}\right\| \leq M$, then $\left\|\mathbf{e}^{n}\right\| \leq M\left\|\mathbf{e}^{0}\right\|$. Hence, $\left\|\mathbf{e}^{n}\right\| \leq\left\|\mathbf{G}^{n}\right\|\left\|\mathbf{e}^{0}\right\|$, $\forall n \in \mathbb{N}$. Consider Equation (18) in the case of $n=1$ and $n \geq 2$, which $\left\|\mathbf{e}^{1}\right\| \leq\left\|\mathbf{e}^{0}\right\|$ if $\left\|\mathbf{G}^{1}\right\| \leq 1$ for $n=1$. In case of $n \geq 2$, this equation can be done by mathematical induction technique. Equation (18) is first rearranged as follows:

$$
\begin{aligned}
\mathbf{e}^{n}= & \mathbf{G}^{n}\left(\mathbf{e}^{n-1}-\frac{1}{\omega_{1}^{(\alpha)}} \sum_{j=2}^{n} \omega_{j}^{(\alpha)}\left(\mathbf{e}^{n-j+1}-\mathbf{e}^{n-j}\right)\right) \\
= & \mathbf{G}^{n}\left(\mathbf{e}^{n-1}-\frac{1}{\omega_{1}^{(\alpha)}} \sum_{j=2}^{n-1} \omega_{j}^{(\alpha)}\left(\mathbf{e}^{n-j+1}-\mathbf{e}^{n-j}\right)-\frac{1}{\omega_{1}^{(\alpha)}} \omega_{n}^{(\alpha)}\left(\mathbf{e}^{1}-\mathbf{e}^{0}\right)\right) \\
= & \mathbf{G}^{n}\left(\left(1-\frac{1}{\omega_{1}^{(\alpha)}} \omega_{2}^{(\alpha)}\right) \mathbf{e}^{n-1}-\frac{1}{\omega_{1}^{(\alpha)}} \sum_{j=3}^{n} \omega_{j}^{(\alpha)} \mathbf{e}^{n-j+1}\right. \\
& \left.+\frac{1}{\omega_{1}^{(\alpha)}} \sum_{j=2}^{n-1} \omega_{j}^{(\alpha)} \mathbf{e}^{n-j}+\frac{\omega_{n}^{(\alpha)}}{\omega_{1}^{(\alpha)}} \mathbf{e}^{0}\right) \\
= & \mathbf{G}^{n}\left(\left(1-\frac{1}{\omega_{1}^{(\alpha)}} \omega_{2}^{(\alpha)}\right) \mathbf{e}^{n-1}-\frac{1}{\omega_{1}^{(\alpha)}} \sum_{j=2}^{n-1} \omega_{j+1}^{(\alpha)} \mathbf{e}^{n-j}\right. \\
& \left.+\frac{1}{\omega_{1}^{(\alpha)}} \sum_{j=2}^{n-1} \omega_{j}^{(\alpha)} \mathbf{e}^{n-j}+\frac{\omega_{n}^{(\alpha)}}{\omega_{1}^{(\alpha)}} \mathbf{e}^{0}\right) \\
= & \mathbf{G}^{n}\left(\left(1-\frac{1}{\omega_{1}^{(\alpha)}} \omega_{2}^{(\alpha)}\right) \mathbf{e}^{n-1}+\frac{1}{\omega_{1}^{(\alpha)}} \sum_{j=2}^{n-1}\left(\omega_{j}^{(\alpha)}-\omega_{j+1}^{(\alpha)}\right) \mathbf{e}^{n-j}+\frac{\omega_{n}^{(\alpha)}}{\omega_{1}^{(\alpha)}} \mathbf{e}^{0}\right) .
\end{aligned}
$$

Take the norm on both sides of Equation (19) and then apply the triangle inequality to get

$$
\begin{aligned}
\left\|\mathbf{e}^{n}\right\| \leq & \left\|\mathbf{G}^{n}\right\|\left(\left(1-\frac{1}{\omega_{1}^{(\alpha)}} \omega_{2}^{(\alpha)}\right)\left\|\mathbf{e}^{n-1}\right\|\right. \\
& \left.+\frac{1}{\omega_{1}^{(\alpha)}} \sum_{j=2}^{n-1}\left(\omega_{j}^{(\alpha)}-\omega_{j+1}^{(\alpha)}\right)\left\|\mathbf{e}^{n-j}\right\|+\frac{\omega_{n}^{(\alpha)}}{\omega_{1}^{(\alpha)}}\left\|\mathbf{e}^{0}\right\|\right) .
\end{aligned}
$$




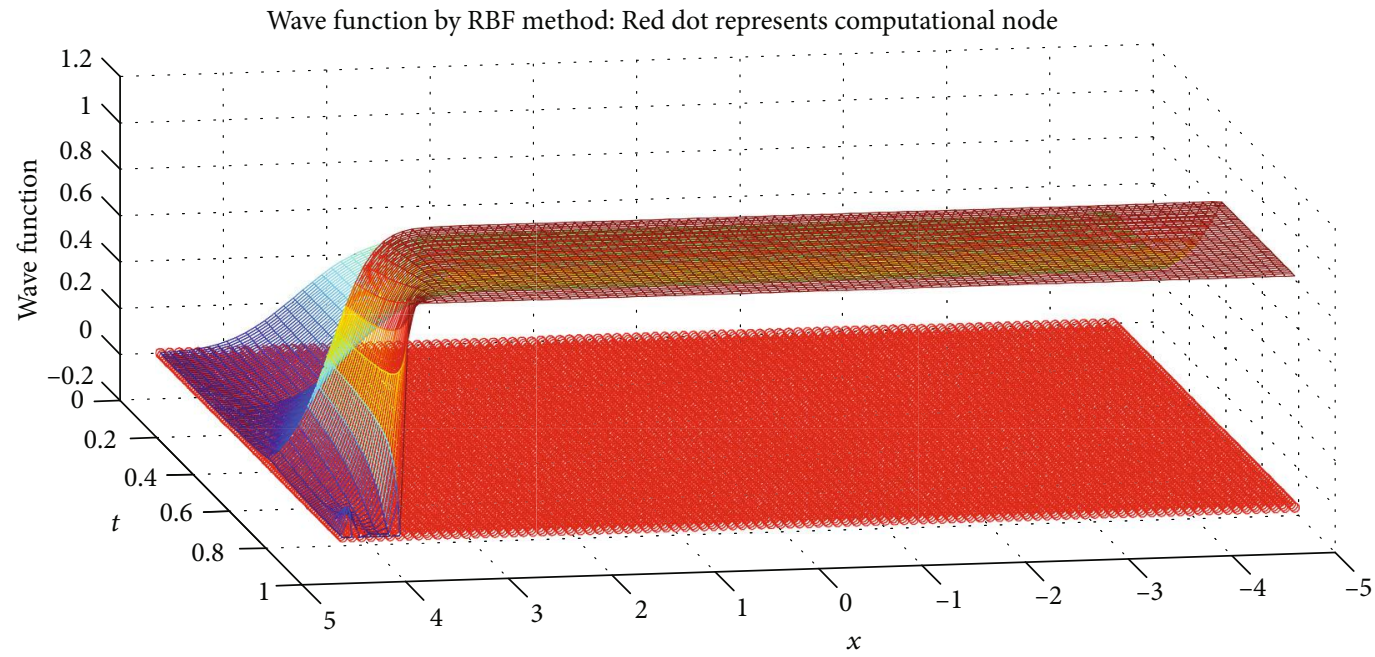

FIgURE 1: The wave function is solved by RBFs method for $\alpha=0.99$.

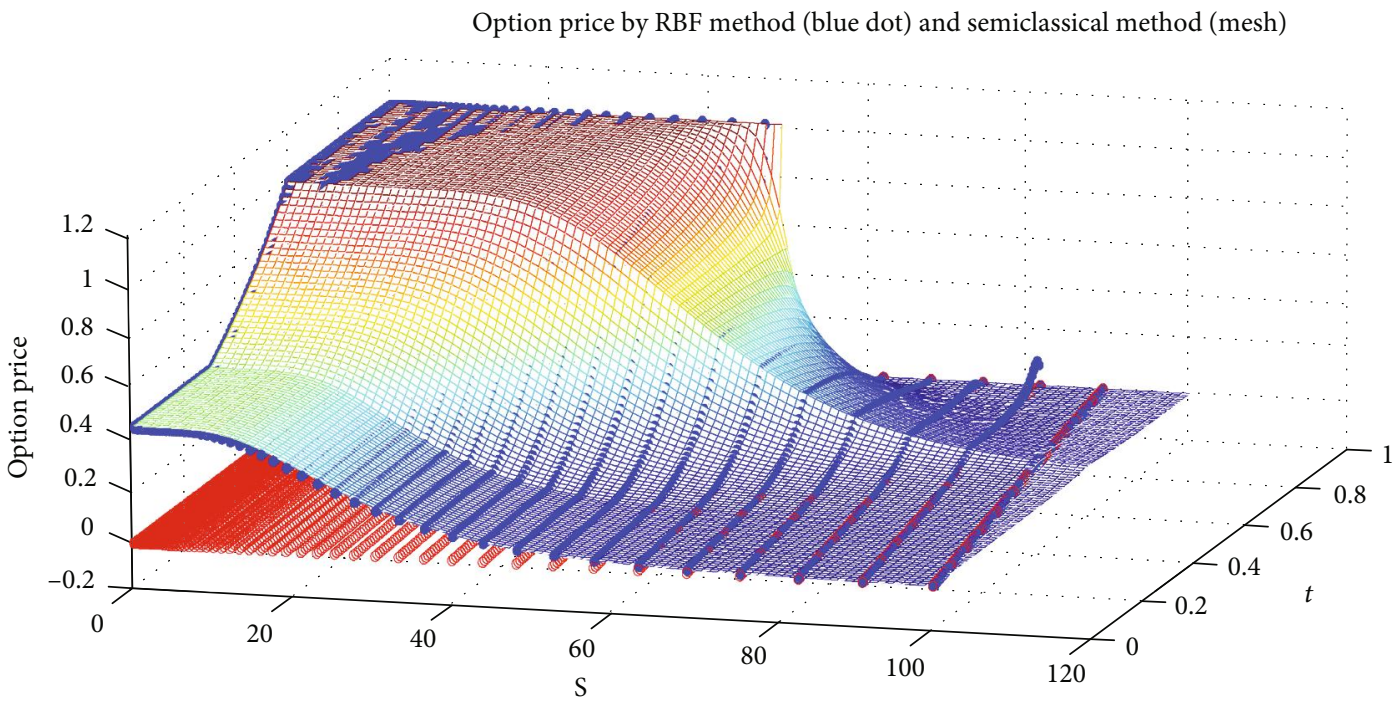

Figure 2: The option price is solved by RBFs method and semiclassical method for $\alpha=0.99$.

For $n=2$, the first step, base case, of mathematical induction intends to give

$$
\begin{aligned}
\left\|\mathbf{e}^{2}\right\| & \leq\left\|\mathbf{G}^{2}\right\|\left(\left(1-\frac{\omega_{2}^{(\alpha)}}{\omega_{1}^{(\alpha)}}\right)\left\|\mathbf{e}^{1}\right\|+\frac{\omega_{2}^{(\alpha)}}{\omega_{1}^{(\alpha)}}\left\|\mathbf{e}^{0}\right\|\right) \\
& \leq\left\|\mathbf{G}^{2}\right\|\left(\left(1-\frac{\omega_{2}^{(\alpha)}}{\omega_{1}^{(\alpha)}}\right)\left\|\mathbf{G}^{1}\right\|\left\|\mathbf{e}^{0}\right\|+\frac{\omega_{2}^{(\alpha)}}{\omega_{1}^{(\alpha)}}\left\|\mathbf{e}^{0}\right\|\right) \\
& <\left\|\mathbf{G}^{2}\right\|\left(\left(1-\frac{\omega_{2}^{(\alpha)}}{\omega_{1}^{(\alpha)}}\right)\left\|\mathbf{e}^{0}\right\|+\frac{\omega_{2}^{(\alpha)}}{\omega_{1}^{(\alpha)}}\left\|\mathbf{e}^{0}\right\|\right) \\
& =\left\|\mathbf{G}^{2}\right\|\left\|\mathbf{e}^{0}\right\|,
\end{aligned}
$$

which is obviously fulfilled. The second step, the inductive case, is proved that $\left\|\mathbf{e}^{n}\right\| \leq\left\|\mathbf{G}^{n}\right\|\left\|\mathbf{e}^{0}\right\|$ for any positive inte- ger. Supposed that $\left\|\mathbf{e}^{m}\right\| \leq\left\|\mathbf{G}^{m}\right\|\left\|\mathbf{e}^{0}\right\|, \forall m \geq 2$ and a term of $\left\|\mathbf{e}^{m+1}\right\| \leq\left\|\mathbf{G}^{m+1}\right\|\left\|\mathbf{e}^{0}\right\|$ is obtained. From an inequality (Equation (20)), it can be written as

$$
\begin{aligned}
\left\|\mathbf{e}^{m+1}\right\| \leq & \left\|\mathbf{G}^{m+1}\right\|\left(\left(1-\frac{\omega_{2}^{(\alpha)}}{\omega_{1}^{(\alpha)}}\right)\left\|\mathbf{e}^{m}\right\|\right. \\
& \left.+\frac{1}{\omega_{1}^{(\alpha)}} \sum_{j=2}^{m}\left(\omega_{j}^{(\alpha)}-\omega_{j+1}^{(\alpha)}\right)\left\|\mathbf{e}^{m-j+1}\right\|+\frac{\omega_{m+1}^{(\alpha)}}{\omega_{1}^{(\alpha)}}\left\|\mathbf{e}^{0}\right\|\right) \\
\leq & \left\|\mathbf{G}^{m+1}\right\|\left(\left(1-\frac{\omega_{2}^{(\alpha)}}{\omega_{1}^{(\alpha)}}\right)\left\|\mathbf{G}^{m}\right\|\left\|\mathbf{e}^{0}\right\|\right. \\
& \left.+\frac{1}{\omega_{1}^{(\alpha)}} \sum_{j=2}^{m}\left(\omega_{j}^{(\alpha)}-\omega_{j+1}^{(\alpha)}\right)\left\|\mathbf{G}^{m-j+1}\right\|\left\|\mathbf{e}^{0}\right\|+\frac{\omega_{m+1}^{(\alpha)}}{\omega_{1}^{(\alpha)}}\left\|\mathbf{e}^{0}\right\|\right)
\end{aligned}
$$




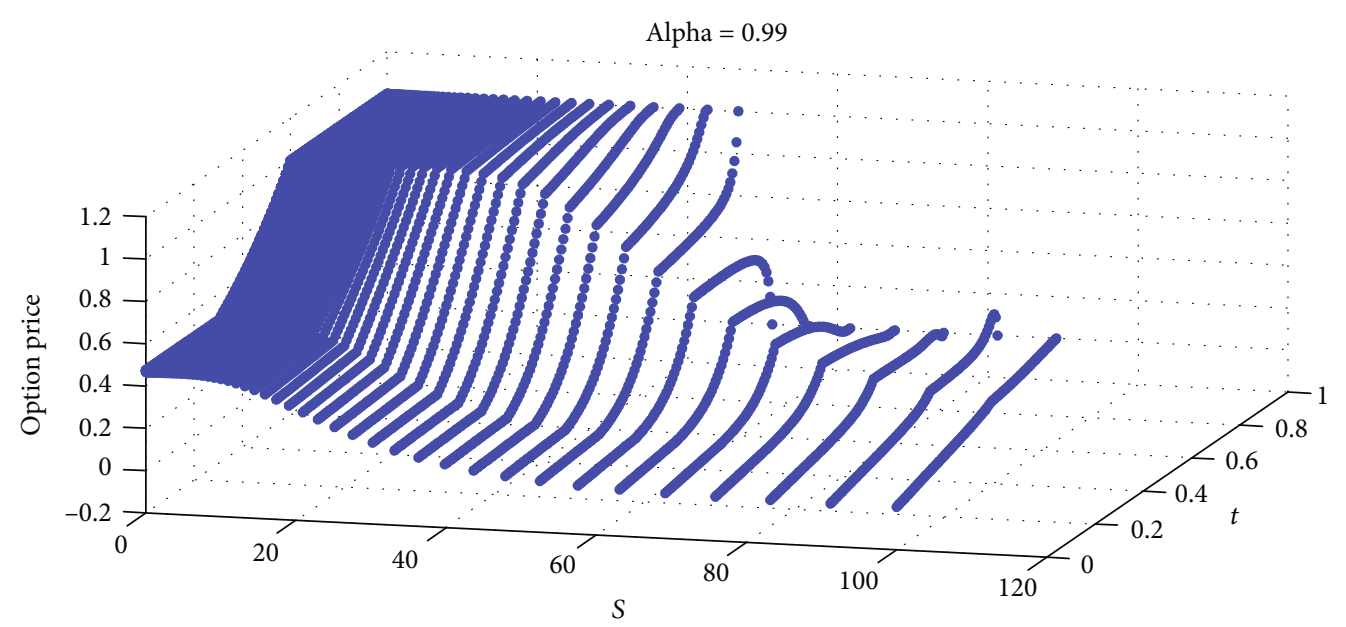

(a)

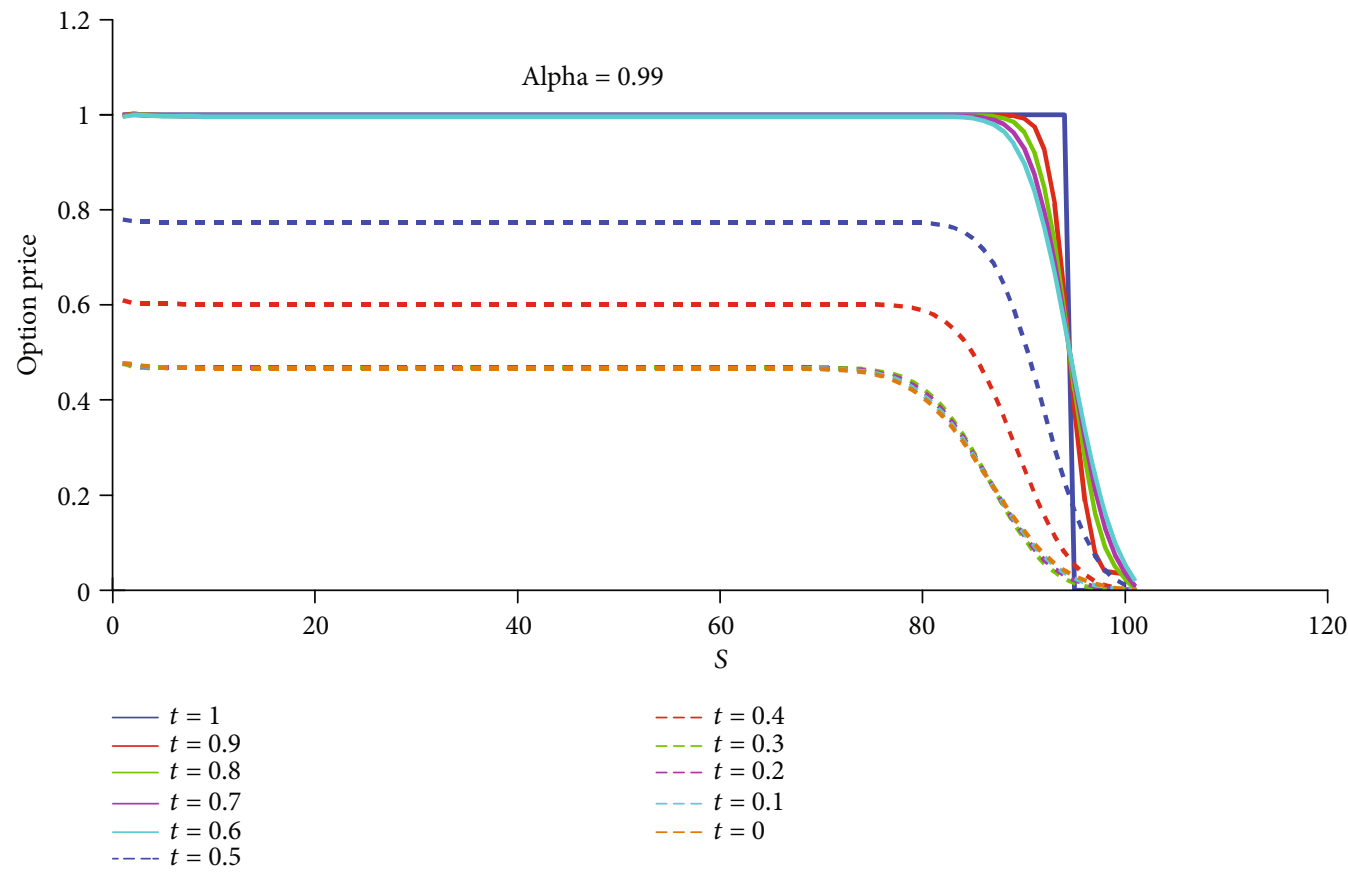

(b)

FIgURE 3: The option price is solved by RBFs method for $\alpha=0.99$.

$$
\begin{aligned}
& <\left\|\mathbf{G}^{m+1}\right\|\left(\left(1-\frac{\omega_{2}^{(\alpha)}}{\omega_{1}^{(\alpha)}}\right)\left\|\mathbf{e}^{0}\right\|\right. \\
& \left.+\frac{1}{\omega_{1}^{(\alpha)}} \sum_{j=2}^{m}\left(\omega_{j}^{(\alpha)}-\omega_{j+1}^{(\alpha)}\right)\left\|\mathbf{G}^{m-j+1}\right\|\left\|\mathbf{e}^{0}\right\|+\frac{\omega_{m+1}^{(\alpha)}}{\omega_{1}^{(\alpha)}}\left\|\mathbf{e}^{0}\right\|\right) \\
& =\left\|\mathbf{G}^{m+1}\right\|\left\|\mathbf{e}^{0}\right\|,
\end{aligned}
$$

which completes the proof. The proof from Equation (22) shows that the errors made at each time level of calculation will be no more than the errors made in the initial step as long as $\left\|\mathbf{G}^{n}\right\| \leq 1$. Since $\mathbf{G}^{n}$ contains a part of matrix $\boldsymbol{A}$ and $\boldsymbol{B}$, their parameter was chosen to satisfy the condition of $\left\|\mathbf{G}^{n}\right\| \leq 1$.

\section{Numerical Experiments and Results}

This section consists of a time step arbitrage bubble and time linear arbitrage bubble cases obtained from [3]. Both cases are represented for confirming the accuracy of the proposed numerical method, since the fractional BlackScholes-Schrodinger equation has no analytical solution. Therefore, the solution of fractional Black-ScholesSchrodinger equation is verified by comparing with the semiclassical solution given by [3]. The semiclassical 


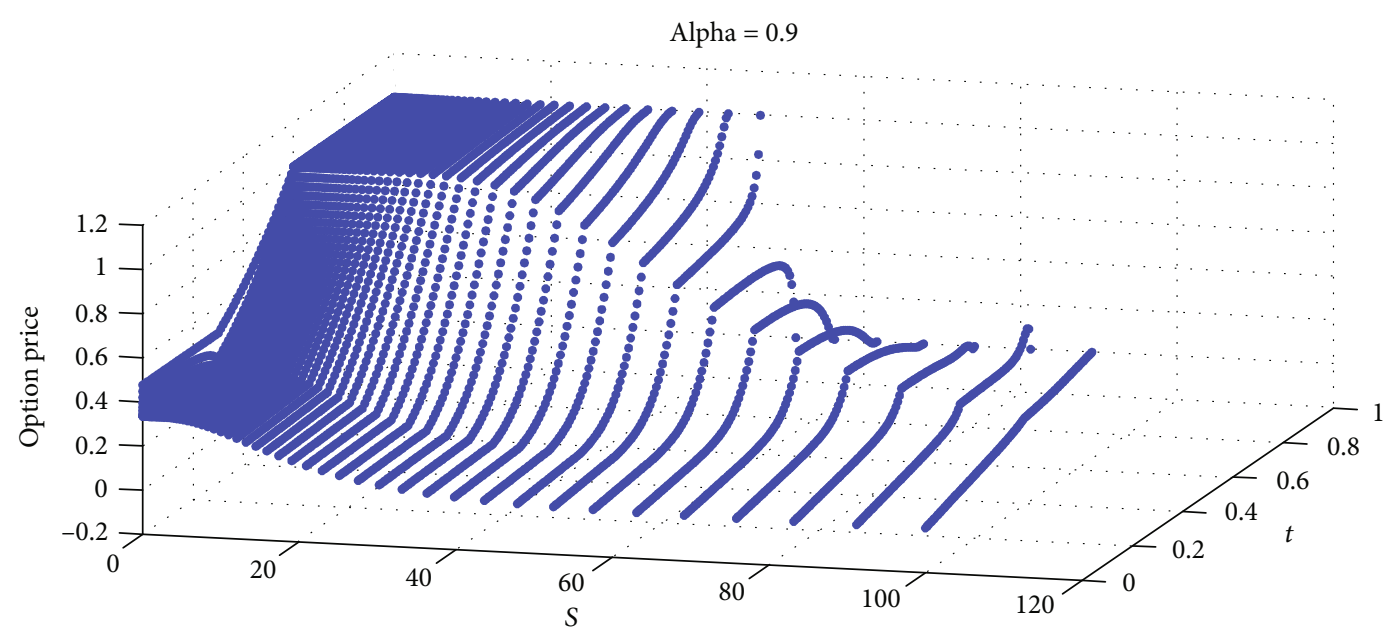

(a)

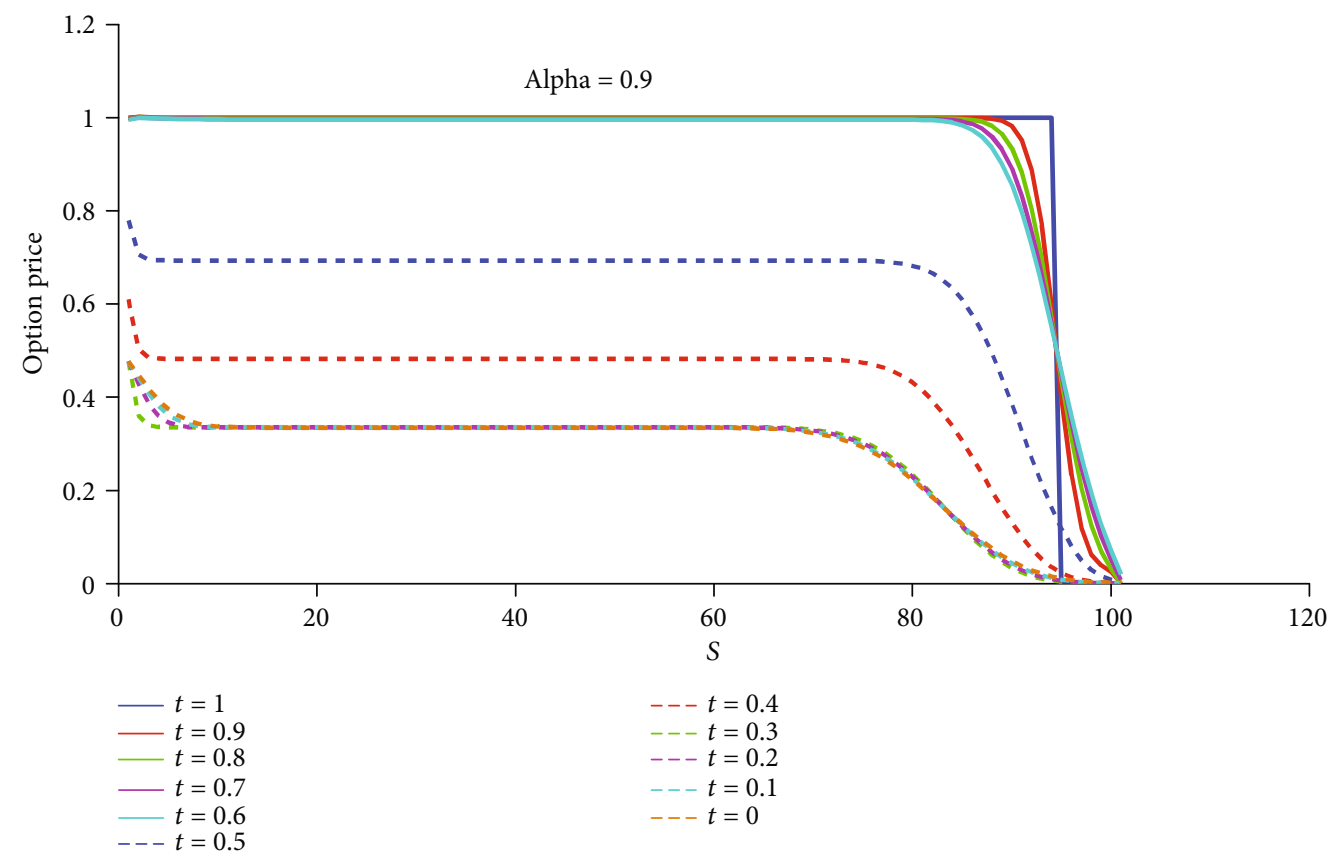

(b)

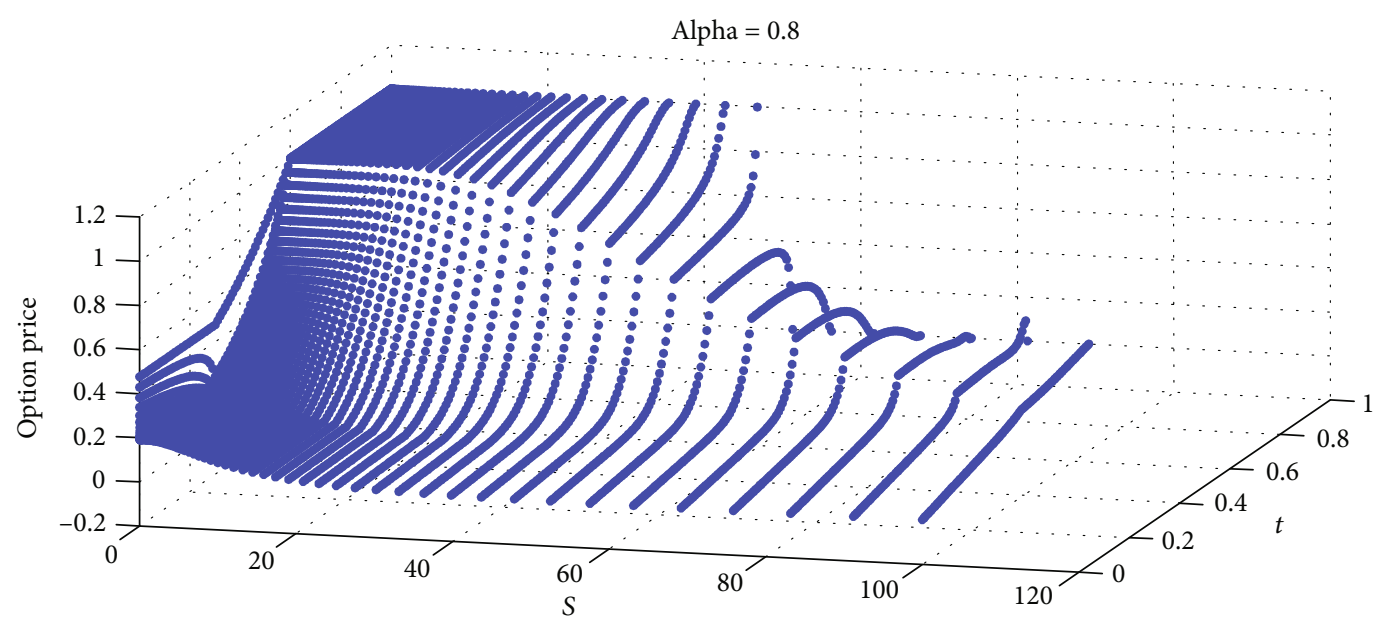

(c)

Figure 4: Continued. 


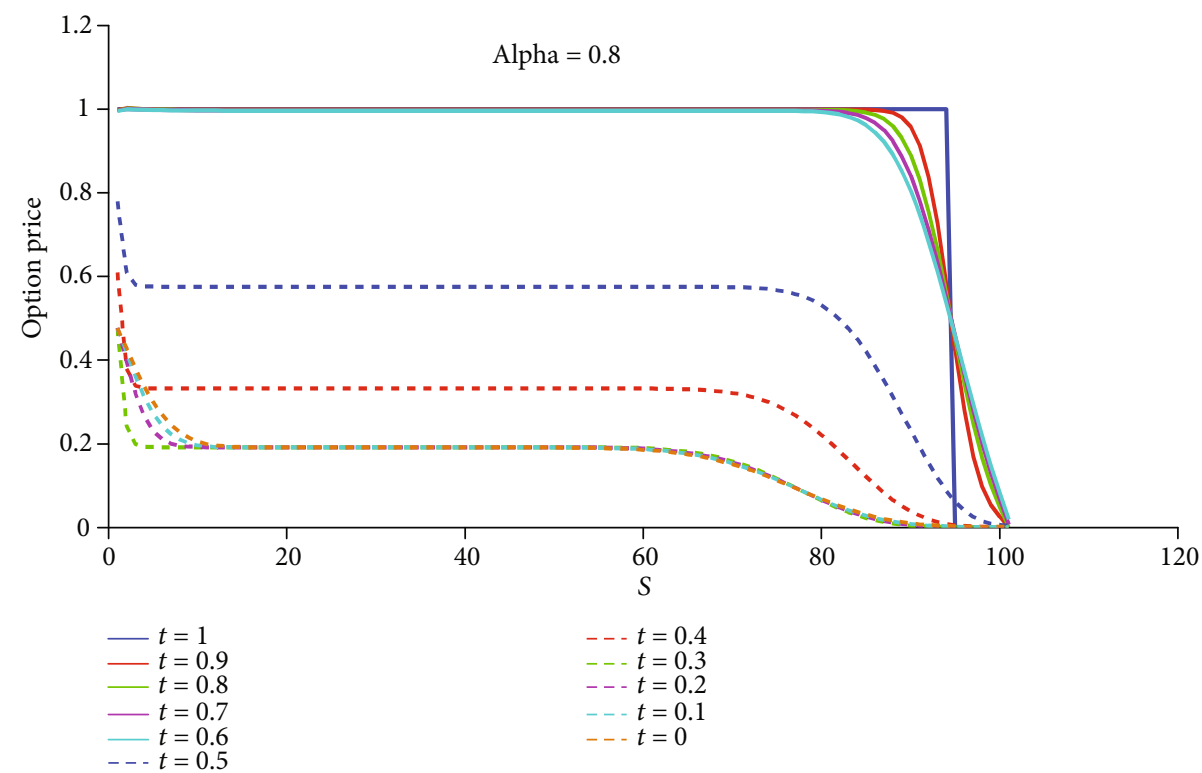

(d)

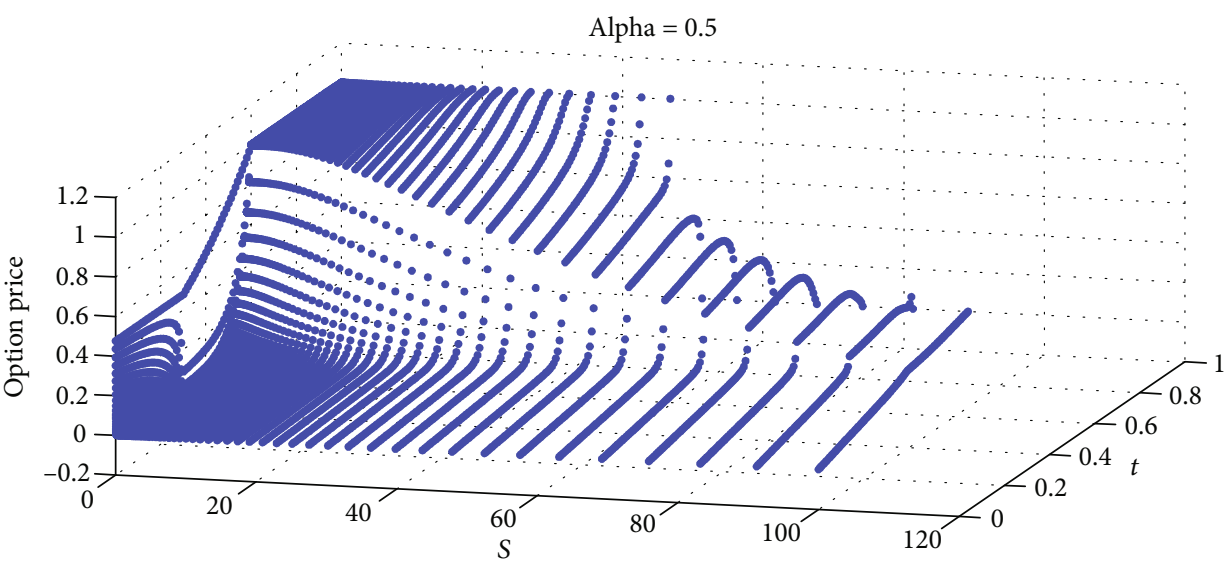

(e)

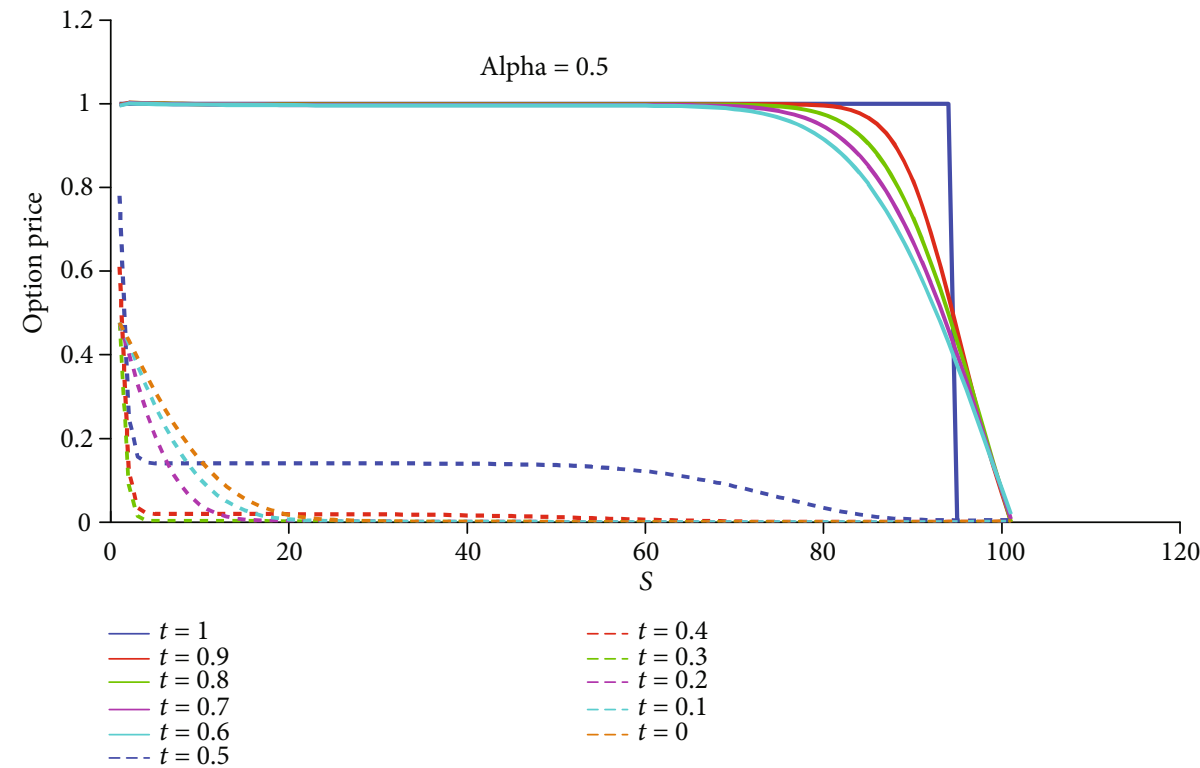

(f)

Figure 4: Continued. 


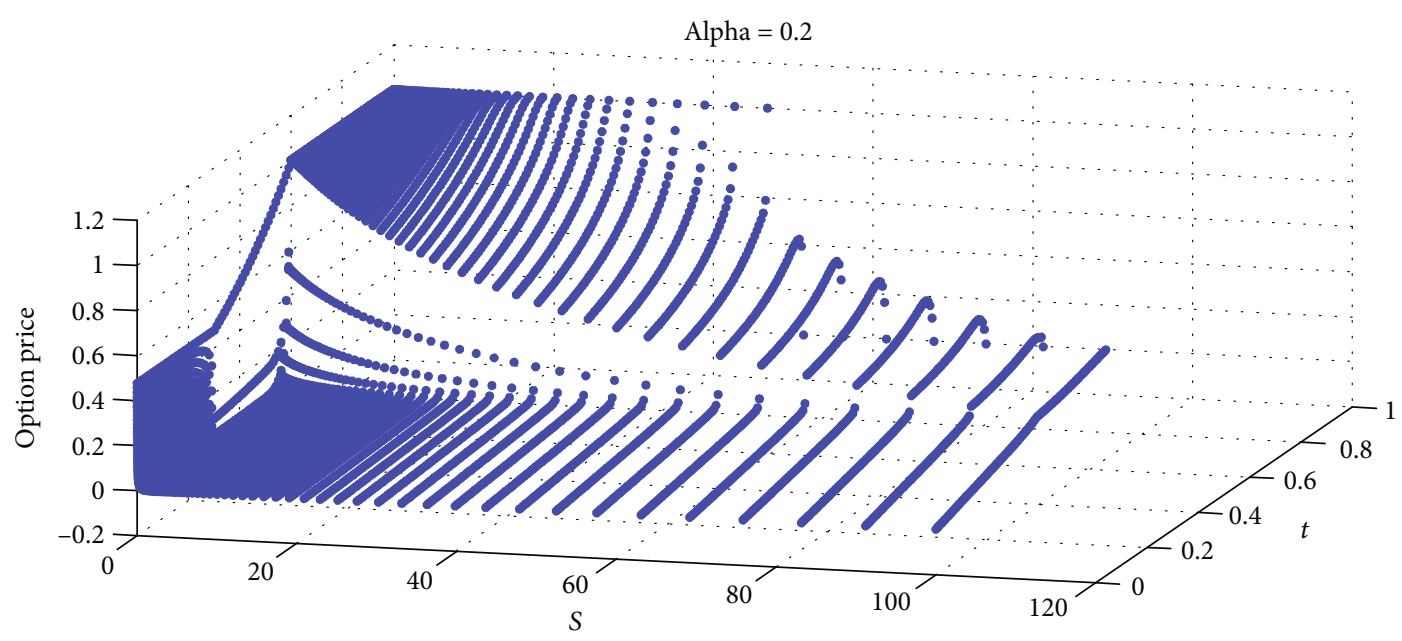

(g)

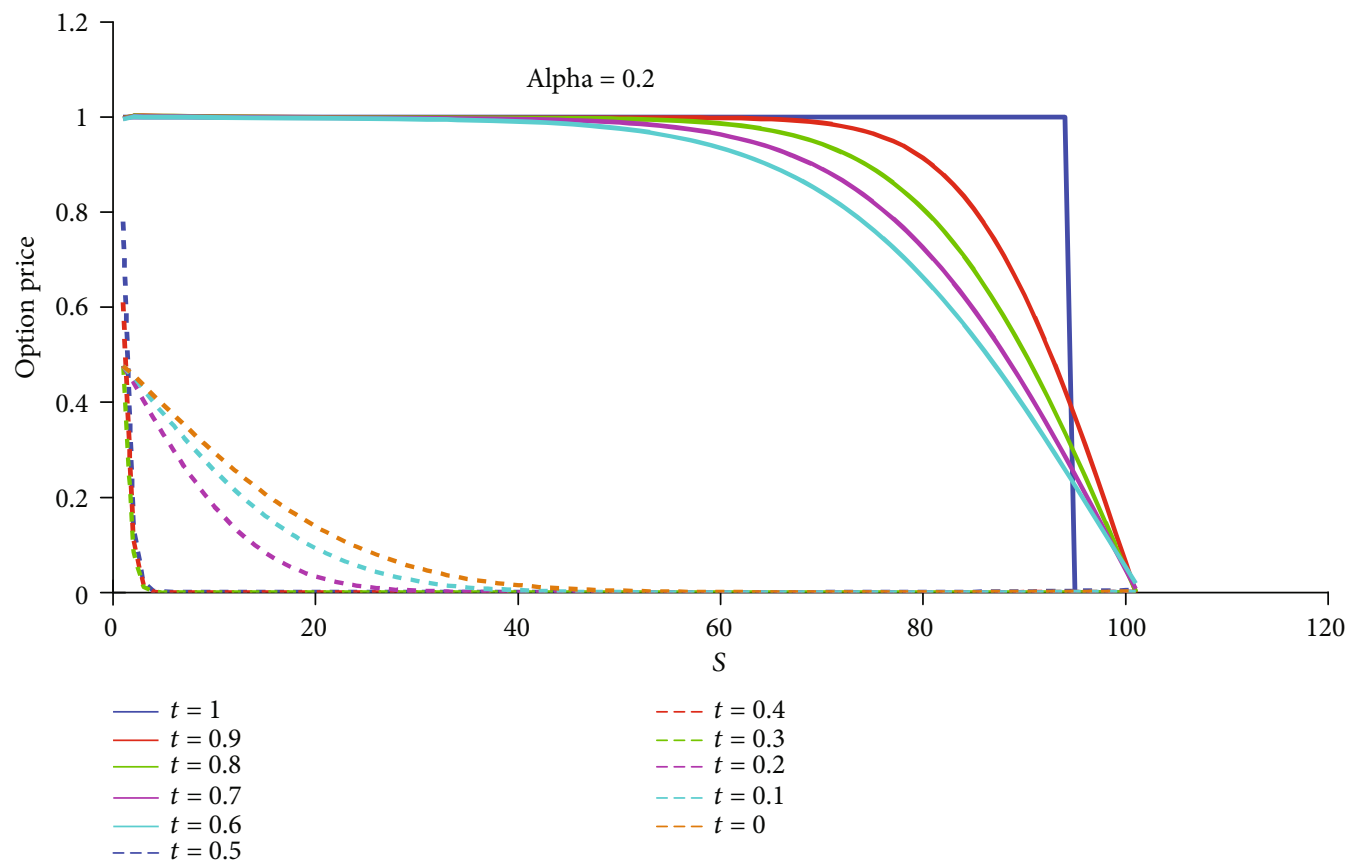

(h)

FIGURE 4: The option price is solved by RBFs method for $\alpha=0.9,0.8,0.5$, and 0.2 .

solution in the presence of a time-dependent arbitrage bubble $f=f(t)$ can be computed as

$$
\pi_{s c}(S, t)=\frac{1}{e^{\rho(t, T)}} \pi_{B S}\left(e^{\rho(t, T)} S, t\right)
$$

where $\pi_{B S}(S, t)$ is the arbitrage-free Black-Scholes solution for the specific option with contract, $\Phi(S)$, and $\rho(t, T)$ is the $\rho$ factor. The arbitrage bubble function, $f$, takes part of the $\rho$ function. In this way, the function $\rho(t, T)$ renormalizes the bare arbitrage-free Black-Scholes solution. The pure Black-Scholes solution $\pi_{B S}(S, t)$ is given by

$$
\pi_{B S}(S, t)=e^{-r(T-t)}\left[1-N\left(d_{2}(S, t)\right)\right],
$$

where $N(x)$ is the normal distribution function and $d_{2}$ $(S, t)=\left(\ln (S / K)+\left(r-\left(\sigma^{2} / 2\right)\right)(T-t)\right) / \sigma \sqrt{(T-t)}$, with a strike price, $K$. The contract function, $\Phi(S)$, is given by

$$
\Phi(S)= \begin{cases}1, & 0<S<K \\ 0, & K<S\end{cases}
$$

The fractional Black-Scholes-Schrodinger equation in the domain $(x, t) \in \mathbb{R} \times[0, T]$ is considered as

$$
\frac{\partial^{\alpha} \psi(x, t)}{\partial t^{\alpha}}+\frac{\sigma^{2}}{2} \frac{\partial^{2} \psi(x, t)}{\partial x^{2}}+v(x, t)\left(\frac{\partial \psi(x, t)}{\partial x}-\psi(x, t)\right)=0
$$




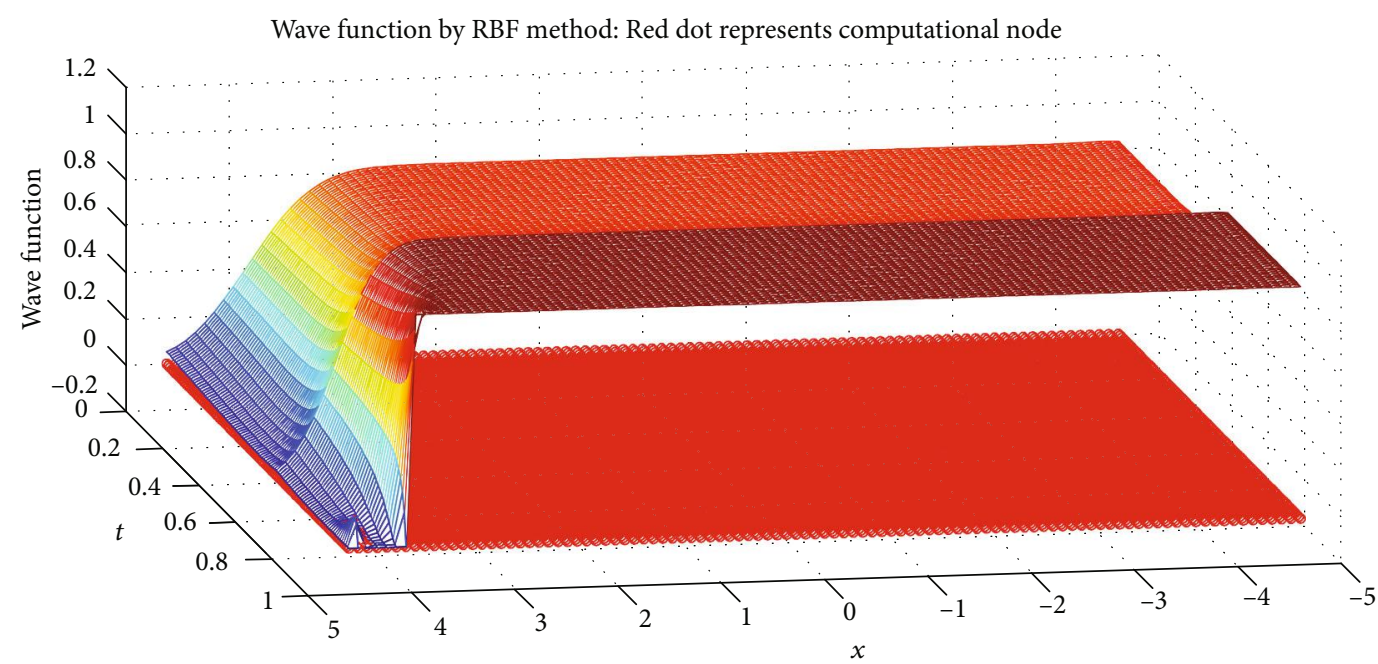

Figure 5: The wave function is solved by RBFs method for $\alpha=0.99$.

Option price by RBF method (bule dot), and semiclassical method (mesh): Red dot represents computational node

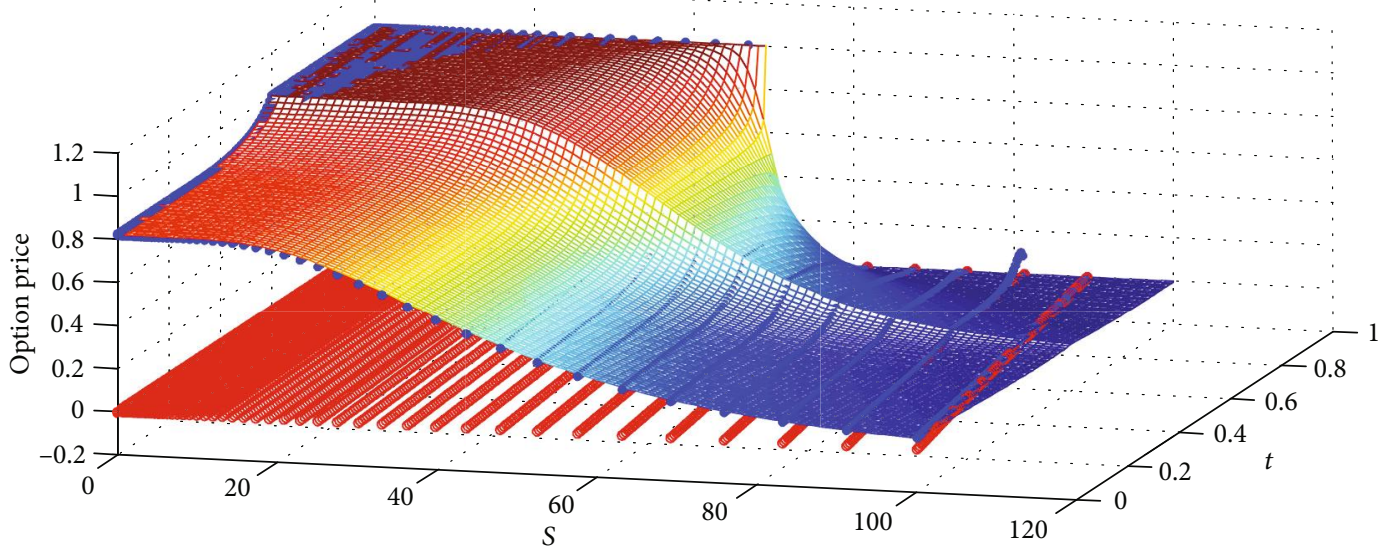

Figure 6: The option price is solved by RBFs method and semiclassical method for $\alpha=0.99$.

The initial and boundary conditions can be obtained by changing variables of the contract function (Equation (25)) and the analytical solution (Equation (23)). Therefore, the initial and boundary conditions are as follows:

$$
\begin{gathered}
\psi(x, T)=\left\{\begin{array}{cc}
1, & -\infty<x<\ln K-\left(r-\frac{1}{2} \sigma^{2}\right) T, \\
0, & \ln K-\left(r-\frac{1}{2} \sigma^{2}\right) T<x .
\end{array}\right. \\
\psi\left(x_{1}, t\right)=e^{r(T-t)} \pi_{s c}\left(e^{x_{1}+\left(r-(1 / 2) \sigma^{2}\right) t}, t\right), \\
\psi\left(x_{N_{x}}, t\right)=e^{r(T-t)} \pi_{s c}\left(e^{x_{N_{x}}+\left(r-(1 / 2) \sigma^{2}\right) t}, t\right),
\end{gathered}
$$

where $x_{1}$ and $x_{N_{x}}$ are the end points boundary of the spatial domain.
Example 1. Consider the following time step arbitrage bubble case of

$$
f(t)= \begin{cases}0, & 0<t<T_{1}, \\ H, & T_{1}<t<T_{2}, \\ 0, & T_{2}<t<T,\end{cases}
$$

with $\rho$ factor condition is determined by

$$
\rho(t, T)= \begin{cases}\left(T_{2}-T_{1}\right) \frac{(r-\bar{\alpha}) H}{\sigma-H}, & 0<t<T_{1}, \\ \left(T_{2}-t\right) \frac{(r-\bar{\alpha}) H}{\sigma-H}, & T_{1}<t<T_{2}, \\ 0, & T_{2}<t<T .\end{cases}
$$

In this paper, a binary put option is analyzed. Since the semiclassical solution is an analytical solution, the result 


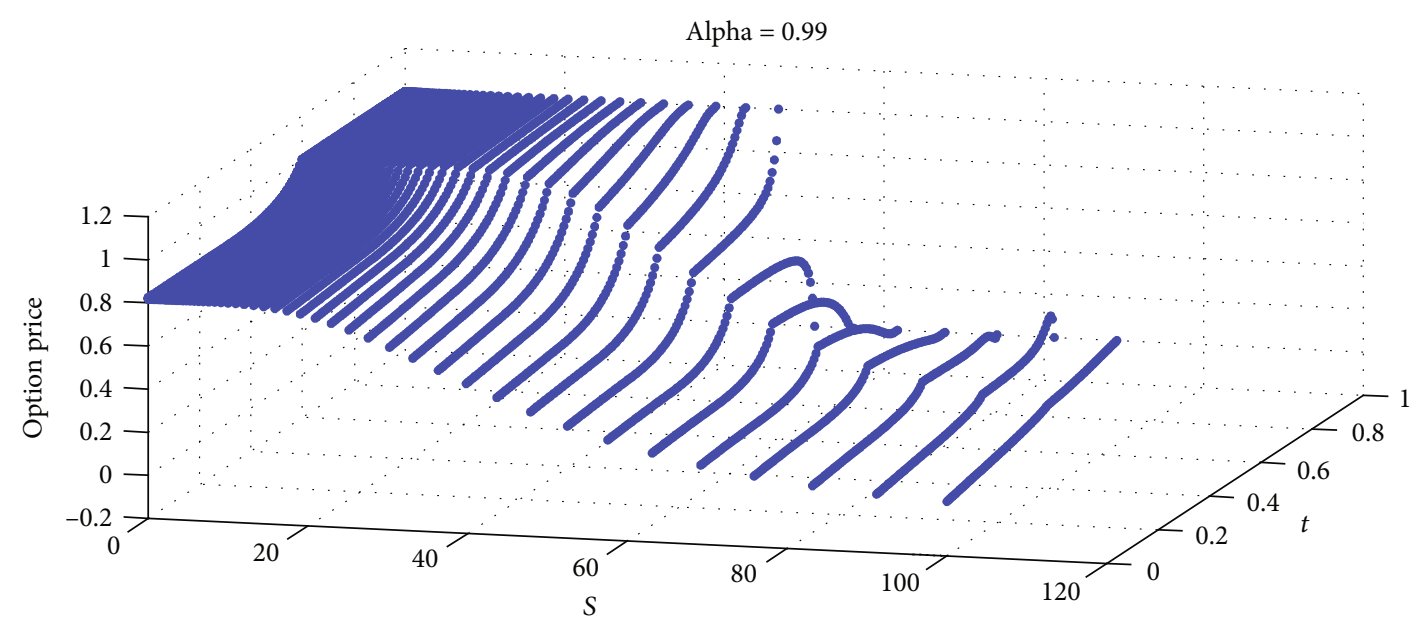

(a)

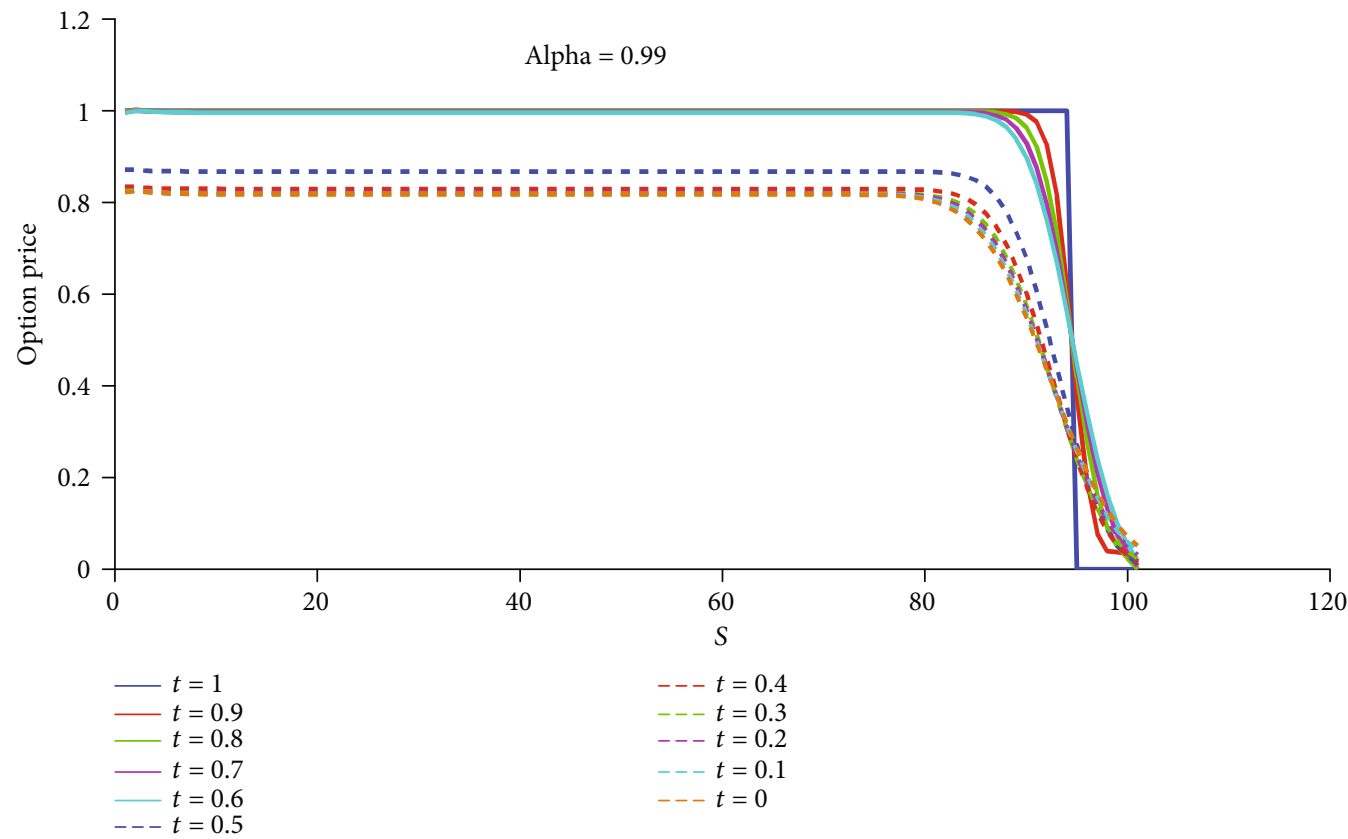

(b)

FIGURE 7: The option price is solved by RBFs method for $\alpha=0.99$.

can be computed from any point on a mesh. However, the aim of this paper is to compare the RBFs solution with semiclassical solution. Accordingly, semiclassical solution that is on the same node with RBFs solution was chosen. A set of node is defined as $N_{x}=100$ and $N_{t}=100$ where $N_{x}$ is the number of support nodes in the interpolation domain of point $x$, and $N_{t}$ is a number of time step. In Example 1, the parameter values are $\alpha=0.99, \sigma=0.5, r=$ $0.01, \bar{\alpha}=-0.6, T_{1}=0.3, T_{2}=0.6, T=1$, and $H=0.1 \sigma$, respectively. Figure 1 shows the wave function solved by RBFs method, where dots on $x$ direction represent computational node, $t$ direction represents a time level, and vertical direction represent a wave function. Figure 2 shows the option price solved by RBFs method and semiclassical method, where $S$ direction represents an underlying asset price, $t$ direction represents a time level, and vertical direction represents the option price. The option price from RBFs method is shown as dots, while the semiclassical solution is shown as a mesh. As it is shown in Figure 2, the dots almost overlapped with the mesh; as a result, the option price of fractional Black-Scholes-Schrodinger equation (in case of $\alpha$ close to 1 ) from RBFs method satisfied the semiclassical solution. On the other hand, Figure 3(a) only shows the option price solved by RBFs method with the initial and boundary conditions; a graph of option price remains steady at the initial time. The option price decrease continuously over time in each time step and remains steady in the final of the graph. In 


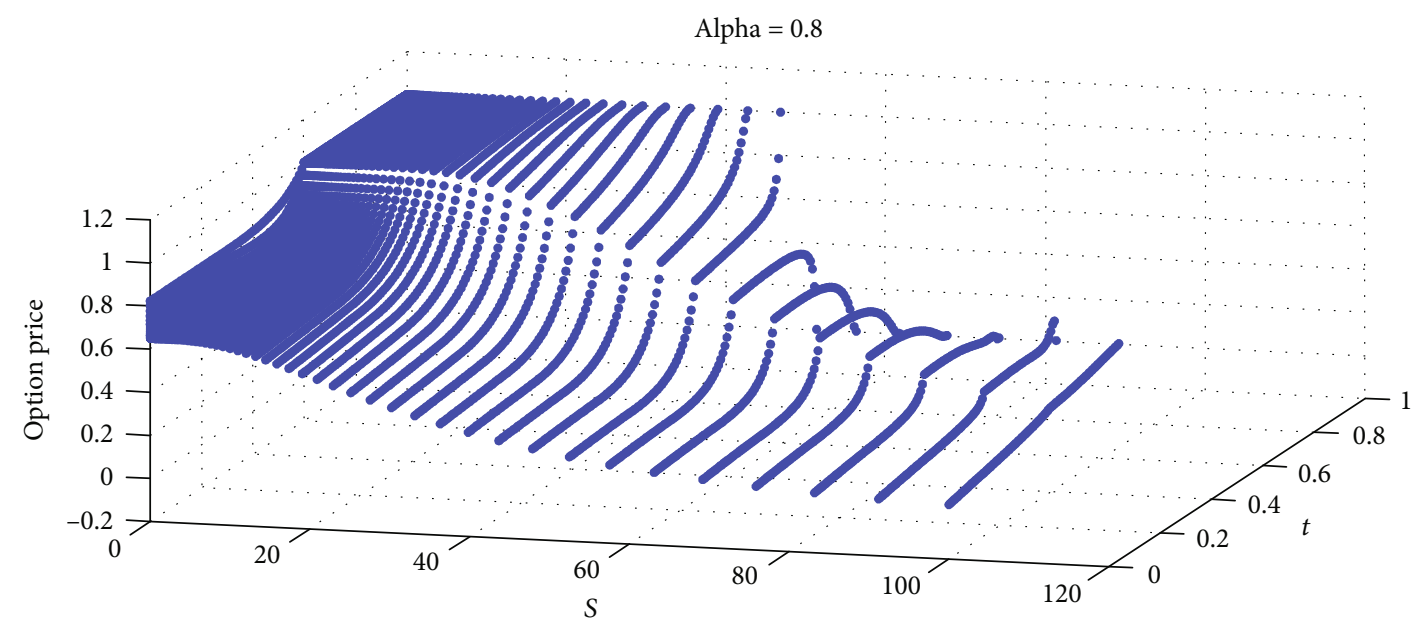

(a)

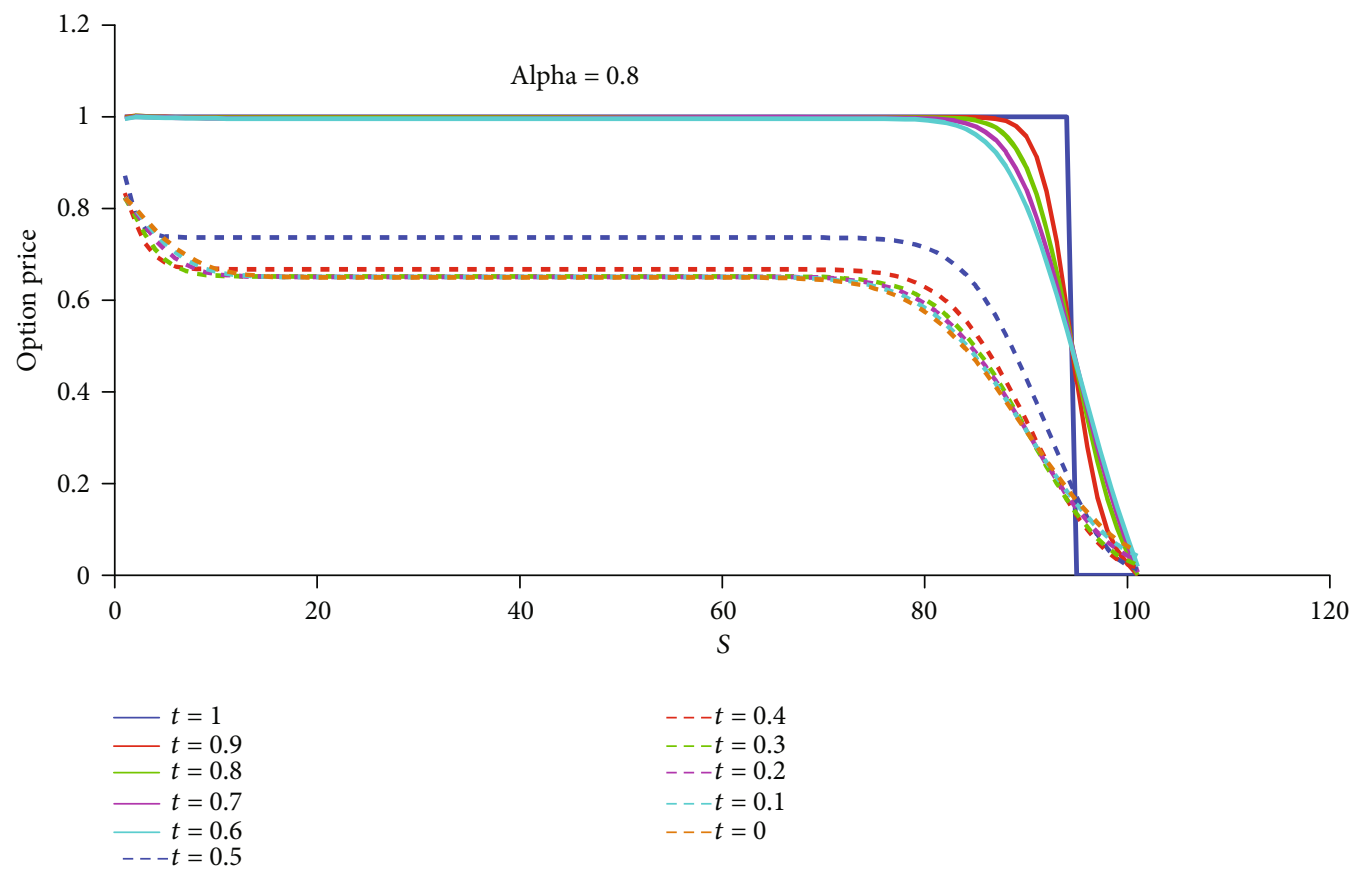

(b)

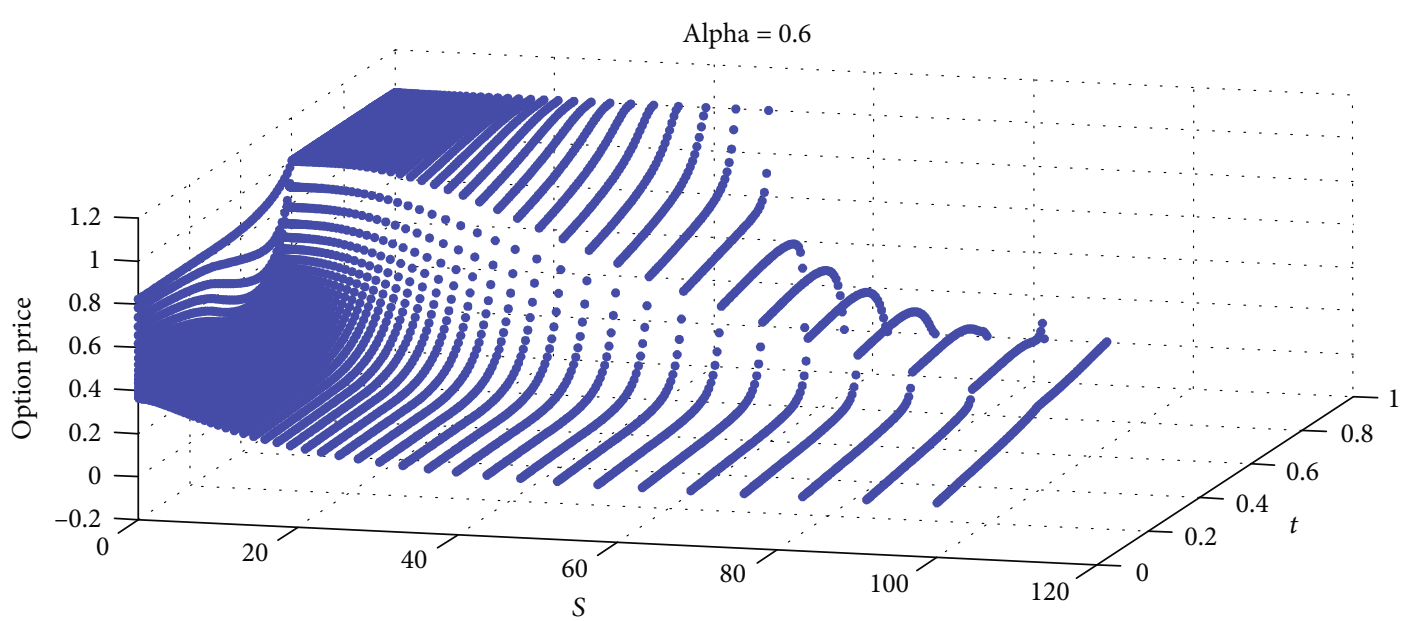

(c) 


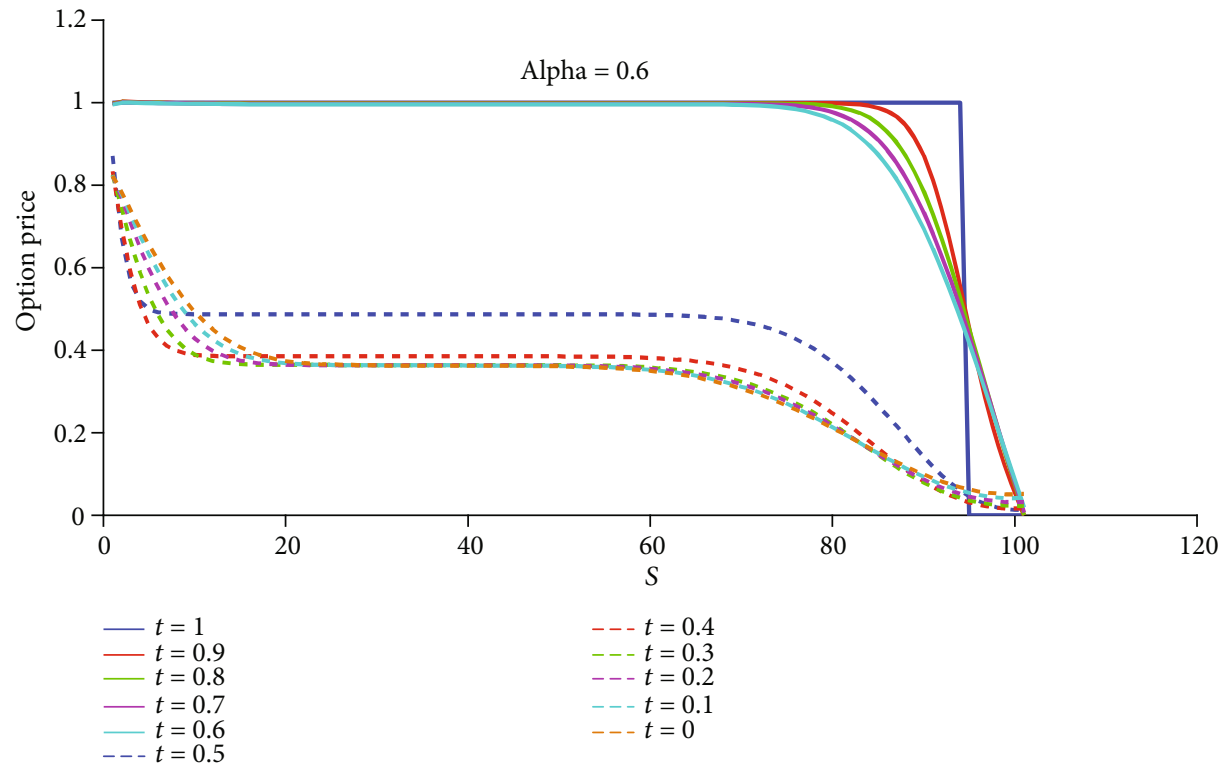

(d)

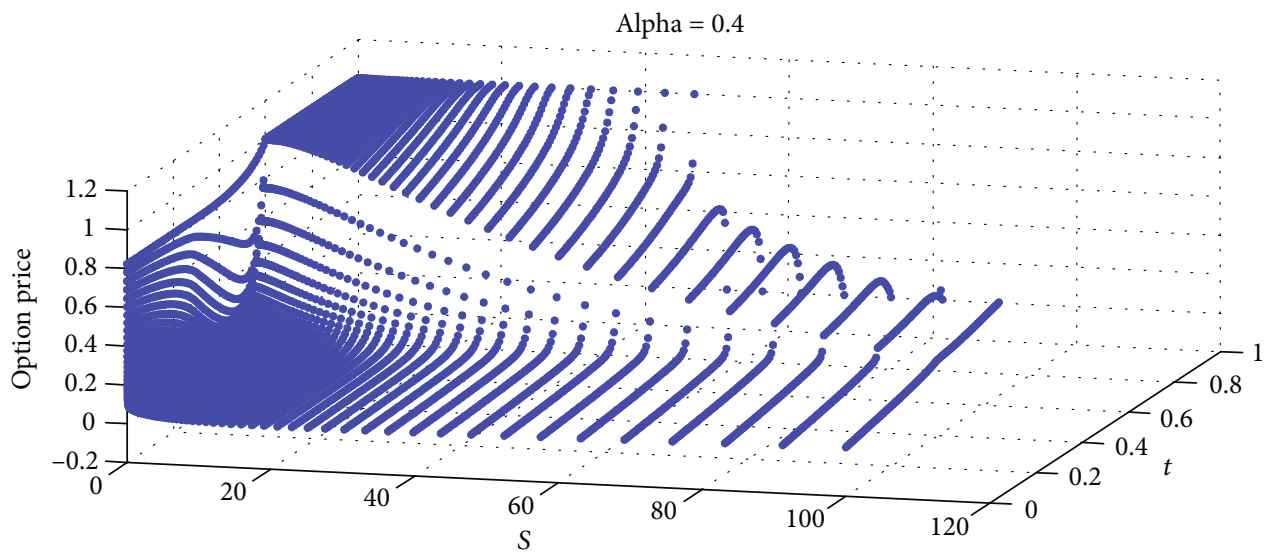

(e)

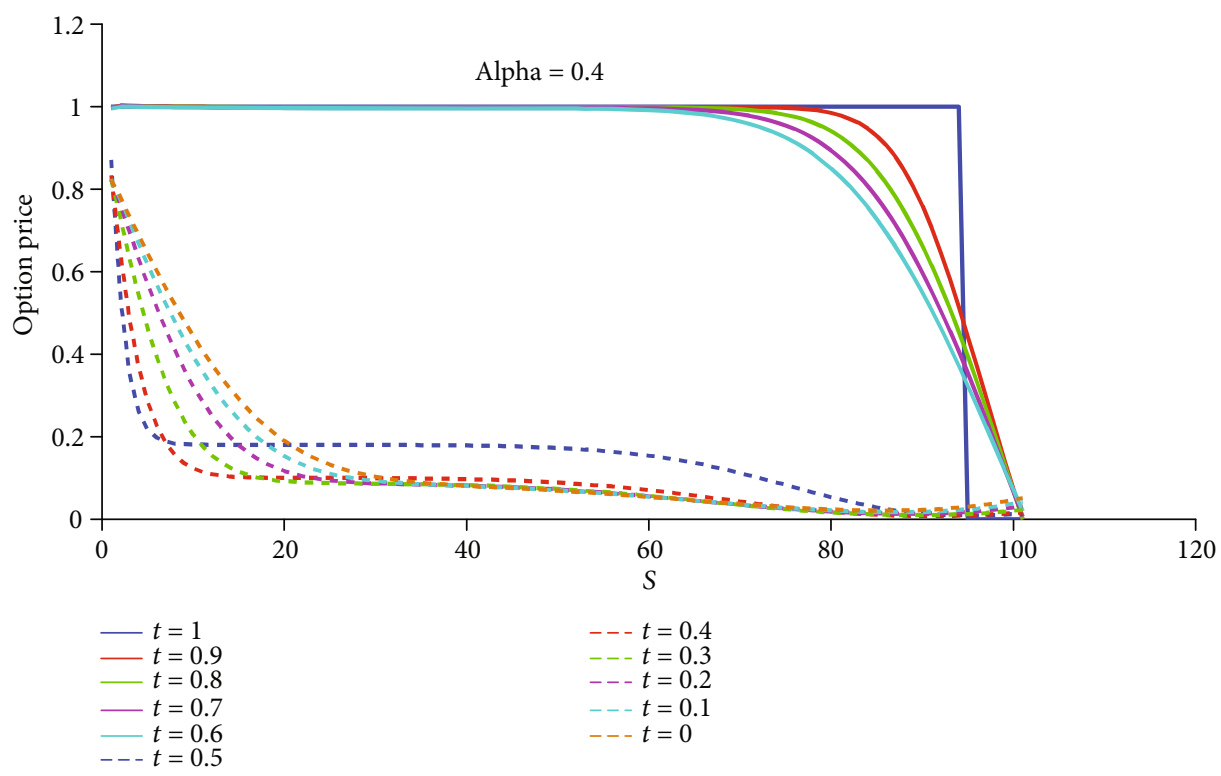

(f)

Figure 8: Continued. 


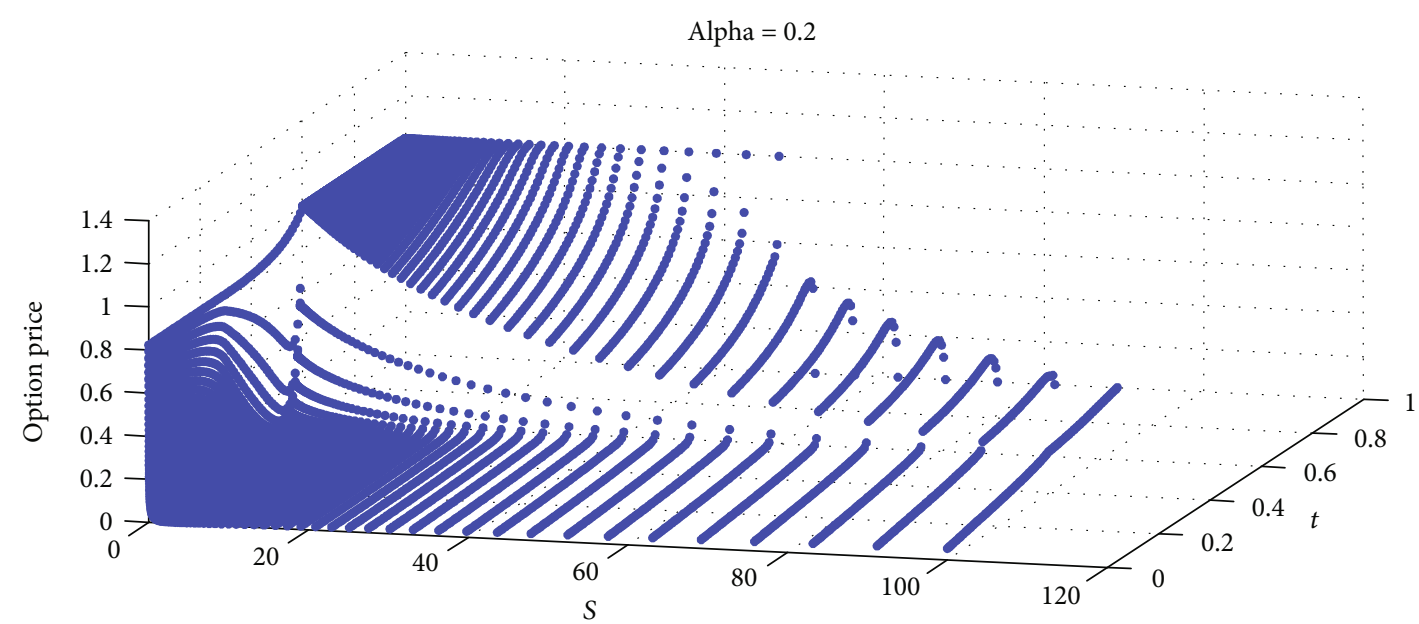

(g)

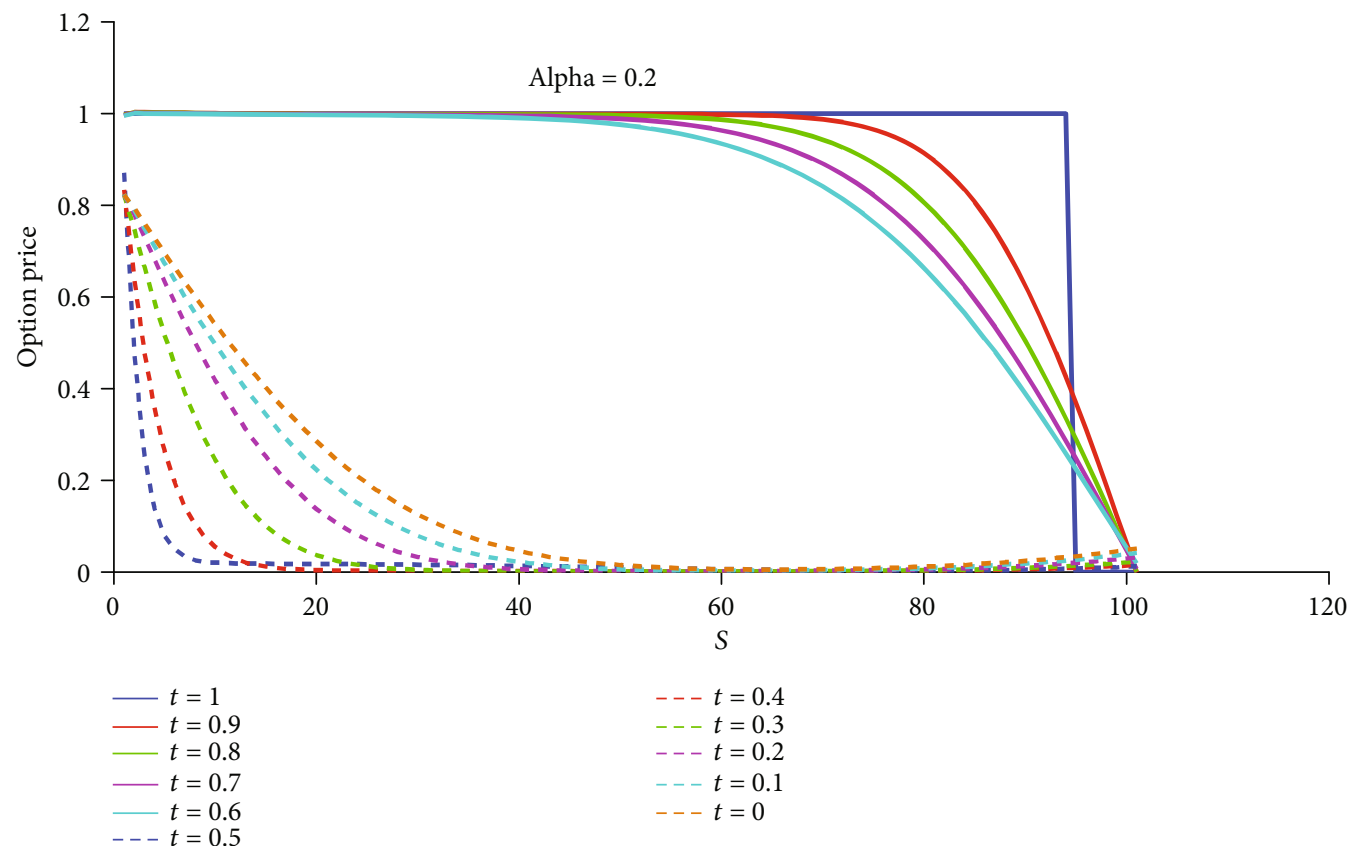

(h)

FIGURE 8: The option price is solved by RBFs method for $\alpha=0.8,0.6,0.4$, and 0.2 .

Figure 3(b), the option price plotted at time 1.0, 0.9, 0.8, 0.7 , and 0.6 shows no difference compared with the graph at time $0.5,0.4$, and 0.3 which show a huge different. Figure 4 shows the option price from RBFs method when the $\alpha$ values are varied. The result shows that the option price decrease more rapidly with the lower $\alpha$ values; for example, the option price at $\alpha=0.2$ decrease with higher slope than the option price at $\alpha=0.5$. Moreover, the $L^{2}$ relative error was analyzed in this paper. The values of the $L^{2}$ relative error between the RBFs solution of fractional Black-Scholes-Schrodinger equation with the semiclassical solution is $9.8544 \times 10^{-3}$. It was found that the numerical result agrees as the semiclassical solution.
In the following example, the option price of the fractional model by RBFs method for time linear arbitrage bubble case is examined.

Example 2. Consider the following time linear arbitrage bubble case

$$
f(t)= \begin{cases}0, & 0<t<T_{1}, \\ \frac{H}{\left(T_{2}-T_{1}\right)}\left(t-T_{1}\right), & T_{1}<t<T_{2}, \\ 0, & T_{2}<t<T,\end{cases}
$$


with $\rho$ factor condition is determined by

$$
\rho(t, T)= \begin{cases}\frac{(r-\bar{\alpha})\left(T_{2}-T_{1}\right)}{H}\left[\sigma \ln \left(\frac{\sigma}{\sigma-H}\right)-H\right], & 0<t<T_{1}, \\ \frac{(r-\bar{\alpha})\left(T_{2}-T_{1}\right)}{H}\left[\sigma \ln \left(\frac{H\left(t-T_{1}\right)-\sigma\left(T_{2}-T_{1}\right)}{\left(T_{2}-T_{1}\right)(H-\sigma)}\right)-\frac{H\left(T_{2}-t\right)}{T_{2}-T_{1}}\right], & T_{1}<t<T_{2}, \\ 0, & T_{2}<t<T .\end{cases}
$$

According to Example 1, a set of node is defined as $N_{x}=100$ and $N_{t}=100$ where $N_{x}$ is the number of support nodes in the interpolation domain of point $x$, and $N_{t}$ is a number of time step. In Example 2, the parameter values are $\alpha=0.99, \sigma=0.5, r=0.01, \bar{\alpha}=-0.6, T_{1}=0.3, T_{2}=0.6$, $T=1$, and $H=0.1 \sigma$, respectively. Figure 5 shows the wave function solved by RBFs method, where dot on $x$ direction represents computational nodes, $t$ direction represents a time level, and vertical direction represents a wave function. Figure 6 shows the option price graph solved by RBFs method and semiclassical method, where $S$ direction represents an underlying asset price, $t$ direction represents a time level, and vertical direction represents the option price which is shown as dots and mesh similar to the graph in previous example. According to Example 1, the dots and mesh almost overlap. Therefore, the option prices of fractional Black-Scholes-Schrodinger equation (in case of $\alpha$ close to 1) by using RBFs method satisfied the semiclassical solution. In contrast, Figure 7(a) only shows the option price solved by RBFs method, a graph of option price remains steady at the initial time. The option price decrease continuously over time in each time step and remains steady in the final of the graph. Figure 7 (b) shows that the option price at times $1.0,0.9,0.8,0.7$, and 0.6 is almost no difference in each time, while at time 0.5 and 0.4 , the option price drops considerably. At time 0.5 and 0.4 , the option price fall slightly at time $0.3,0.2,0.1$, and 0 . Figure 8 shows the option price in different values of $\alpha$. When, $\alpha$ value decreases, the option price changes dramatically. In other hand, the option price changes slightly when the values of $\alpha$ increases. According to Example 1, the values of the $L^{2}$ relative error between the RBFs solution of fractional Black-Scholes-Schrodinger equation with the semiclassical solution is $7.9318 \times 10^{-3}$. It was found that the numerical result agrees as the semiclassical solution.

From previous examples, it concluded that the results from RBFs method (in case of $\alpha$ close to 1) satisfied the semiclassical solution. The results of the fractional model for various $\alpha$ values show that when $\alpha$ value is decreased, the option price is changing rapidly while when $\alpha$ value increases close to 1 , the solutions are changing slowly.

\section{Conclusion}

The numerical method in solving the fractional BlackScholes-Schrodinger equation based on the radial basis func- tions (RBFs) method is successfully developed. The spatial derivative is discretized by the RBFs method. A simple quadrature formula is applied in terms of time fractional derivative discretizing. The Gaussian function is selected as a radial basis function in RBFs method. The numerical solutions of fractional Black-Scholes-Schrodinger equation based on RBFs method in case of fractional order close to 1 is compared with the semiclassical solution. The results presented as time step arbitrage bubble case and time linear arbitrage bubble case, which show that the option price from RBFs method satisfied the semiclassical solution. Therefore, RBFs method can be used to solve the fractional Black-ScholesSchrodinger equation. Because the effect in changing the value of alpha on the fractional model is considered, the option price changes rapidly when the value of alpha is decreased. On the other hands, when alpha was increasing close to 1 , the option price changed slowly. Therefore, it can be concluded that the varying fractional order affected to the model solutions. The value of the $L^{2}$ relative error between the RBFs solution of fractional Black-ScholesSchrodinger equation with the semiclassical solution was analyzed in both example cases. It shows that the numerical result agrees as the semiclassical solution. Furthermore, the RBFs method is verified by the stability analysis. The results of solving the fractional Black-Scholes-Schrodinger equation by applied RBFs method can be ensured by stability analysis. Thus, this study provided an alternative useful approach in solving the fractional Black-Scholes-Schrodinger equation.

\section{Data Availability}

The data used to support the findings of this study are available from the corresponding author upon request.

\section{Conflicts of Interest}

The authors declare that they have no competing interests.

\section{Authors' Contributions}

The idea of this research was introduced by AL and NN. All authors contributed to the main results and numerical simulations. $\mathrm{AL}$ contributed to revising the manuscript. All authors read and approved the final manuscript. 


\section{Acknowledgments}

The authors wish to express their gratitude to the editors and the reviewers for the helpful comments. This research is supported by the Department of Mathematics, Faculty of Science, King Mongkut's University of Technology Thonburi (KMUTT) and Uttaradit Rajabhat University.

\section{References}

[1] F. Black and M. Scholes, "The pricing of options and corporate liabilities," Journal of Political Economy, vol. 81, no. 3, pp. 637654, 1973.

[2] M. Contreras, R. Montalva, R. Pellicer, and M. Villena, "Dynamic option pricing with endogenous stochastic arbitrage," Physica A: Statistical Mechanics and its Applications, vol. 389, no. 17, pp. 3552-3564, 2010.

[3] M. Contreras, R. Pellicer, M. Villena, and A. Ruiz, “A quantum model of option pricing: when Black-Scholes meets Schrödinger and its semi-classical limit," Physica A: Statistical Mechanics and its Applications, vol. 389, no. 23, pp. 5447-5459, 2010.

[4] D. A. Benson, R. Schumer, M. M. Meerschaert, and S. W. Wheatcraft, "Fractional dispersion, Lévy motion, and the MADE tracer tests," in Dispersion in Heterogeneous Geological Formations, pp. 211-240, Springer, Dordrecht, 2001.

[5] D. A. Benson, S. W. Wheatcraft, and M. M. Meerschaert, "Application of a fractional advection-dispersion equation," Water Resources Research, vol. 36, no. 6, pp. 1403-1412, 2000.

[6] Y. Pachepsky, D. Timlin, and W. Rawls, "Generalized Richards' equation to simulate water transport in unsaturated soils," Journal of Hydrology, vol. 272, no. 1-4, pp. 3-13, 2003.

[7] A. Cartea and D. del-Castillo-Negrete, "Fractional diffusion models of option prices in markets with jumps," Physica A: Statistical Mechanics and its Applications, vol. 374, no. 2, pp. 749-763, 2007.

[8] E. Scalas, R. Gorenflo, and F. Mainardi, "Fractional calculus and continuous-time finance," Physica A: Statistical Mechanics and its Applications, vol. 284, no. 1-4, pp. 376-384, 2000.

[9] E. Hanert, E. Schumacher, and E. Deleersnijder, "Front dynamics in fractional-order epidemic models," Journal of Theoretical Biology, vol. 279, no. 1, pp. 9-16, 2011.

[10] S. Das and P. K. Gupta, "A mathematical model on fractional Lotka-Volterra equations," Journal of Theoretical Biology, vol. 277, no. 1, pp. 1-6, 2011.

[11] E. Hanert, "Front dynamics in a two-species competition model driven by Lévy flights," Journal of Theoretical Biology, vol. 300, pp. 134-142, 2012.

[12] N. Thamareerat, A. Luadsong, and N. Aschariyaphotha, "Stability results of a fractional model for unsteady-state fluid flow problem," Advances in Difference Equations, vol. 2017, no. 1, 2017.

[13] M. Caputo, "Linear models of dissipation whose Q is almost frequency independent-II," Geophysical Journal of the Royal Astronomical Society, vol. 13, no. 5, pp. 529-539, 1967.

[14] K. B. Oldham and J. Spanier, The Fractional Calculus, Academic Press, New York, 1974.

[15] K. S. Miller and B. Ross, An Introduction to the Fractional Calculus and Fractional Differential Equations, Wiley, New York, 1993.

[16] I. Podlubny, Fractional Differential Equations, Academic Press, San Diego, 1999.
[17] A. A. Kilbas, H. M. Srivastava, and J. J. Trujillo, Theory and Applications of Fractional Differential Equations, Elsevier, San Diego, 2006.

[18] Z. Cen and A. Le, "A robust and accurate finite difference method for a generalized Black-Scholes equation," Journal of Computational and Applied Mathematics, vol. 235, no. 13, pp. 3728-3733, 2011.

[19] L. Song and W. Wang, "Solution of the fractional BlackScholes option pricing model by finite difference method," Abstract and Applied Analysis, vol. 2013, Article ID 194286, 10 pages, 2013

[20] S. Kumar, D. Kumar, and J. Singh, "Numerical computation of fractional Black-Scholes equation arising in financial market," Egyptian Journal of Basic and Applied Sciences, vol. 1, no. 3-4, pp. 177-183, 2014.

[21] P. Phaochoo, A. Luadsong, and N. Aschariyaphotha, "A numerical study of the European option by the MLPG method with moving kriging interpolation," SpringerPlus, vol. 5, no. 1, p. 305, 2016.

[22] P. Phaochoo, A. Luadsong, and N. Aschariyaphotha, "The meshless local Petrov-Galerkin based on moving kriging interpolation for solving fractional Black-Scholes model," Journal of King Saud University - Science, vol. 28, no. 1, pp. 111-117, 2016.

[23] K. Phramrung, A. Luadsong, and N. Aschariyaphotha, "Numerical approximation of the fractional HIV model using the meshless local Petrov-Galerkin method," Advances in Difference Equations., vol. 2019, no. 1, p. 377, 2019.

[24] Z. Cen, J. Huang, A. Xu, and A. Le, "Numerical approximation of a time-fractional Black-Scholes equation," Computers \& Mathematics with Applications, vol. 75, no. 8, pp. 2874-2887, 2018.

[25] C. Chen, Z. Wang, and Y. Yang, "A new operator splitting method for American options under fractional Black-Scholes models," Computers \& Mathematics with Applications, vol. 77, no. 8, pp. 2130-2144, 2019.

[26] M. Uddin and M. Taufiq, "Approximation of time fractional Black-Scholes equation via radial kernels and transformations," Fractional Differential Calculus, vol. 9, no. 1, pp. 75-90, 2019.

[27] R. L. Hardy, "Multiquadric equations of topography and other irregular surfaces," Journal of Geophysical Research, vol. 76, no. 8, pp. 1905-1915, 1971.

[28] F. S. Zafarghandi, M. Mohammadi, E. Babolian, and S. Javadi, "Radial basis functions method for solving the fractional diffusion equations," Applied Mathematics and Computation, vol. 342, pp. 224-246, 2019.

[29] K. Uhlir and V. Skala, "Reconstruction of damaged images using radial basis functions," in Proceedings of 13 th European Signal Processing Conference (EUSIPCO) 2005, pp. 160-163, Antalya, Turkey, 2005.

[30] J. Zapletal, P. Vaněček, and V. Skala, "RBF-based image restoration utilising auxiliary points," in Proceedings of the 2009 Computer Graphics International Conference, ACM, 2009, pp. 39-43, Victoria British Columbia Canada, May 2009.

[31] R. Pan and V. Skala, "A two-level approach to implicit surface modeling with compactly supported radial basis functions," Engineering with Computers, vol. 27, no. 3, pp. 299-307, 2011.

[32] A. Pena, "Option pricing with radial basis functions: a tutorial,” Technical report, Wilmott Magazine, 2005. 
[33] Y. C. Hon and X. Z. Mao, "A radial basis function method for solving options pricing model," Financial Engineering, vol. 8, no. 1, pp. 31-49, 1999.

[34] Y. C. Hon, "A quasi-radial basis functions method for American options pricing," Computers and Mathematics with Applications, vol. 43, no. 3-5, pp. 513-524, 2002.

[35] S. Zhang, "Radial basis functions method for valuing options: a multinomial tree approach," Journal of Computational and Applied Mathematics, vol. 319, pp. 97-107, 2017.

[36] Q. Liu, Y. T. Gu, P. Zhuang, F. Liu, and Y. F. Nie, “An implicit $\mathrm{RBF}$ meshless approach for time fractional diffusion equations," Computational Mechanics, vol. 48, no. 1, pp. 1-12, 2011.

[37] D. A. Murio, "Implicit finite difference approximation for time fractional diffusion equations," Computers and Mathematics with Applications, vol. 56, no. 4, pp. 1138-1145, 2008. 